\title{
Okolište - Spätneolithische Keramik und Siedlungsentwicklung in Zentralbosnien: Ergebnisse und Interpretationen
}

\author{
Robert Hofmann \\ Kiel
}

\section{Einleitung}

Ausgehend von Anatolien und Griechenland breitete sich um etwa 6000 v. u. Z. in Südosteuropa Ackerbau und Viehzucht aus. Das frühe Neolithikum (6100-5500 v. u. Z.) ist zunächst noch durch relativ kleine soziale Gruppen und zahlreiche kleine und eher kurzlebige Siedlungen gekennzeichnet. Ab etwa 5500 v. u. Z. begannen sich erstmals in der Geschichte Südosteuropas große langlebige Dörfer mit teils enormen Bevölkerungskonzentrationen $\mathrm{zu}$ etablieren, die menschliche Gesellschaften in sozialer und wirtschaftlicher Hinsicht vor völlig neue Herausforderungen stellten. Von den primären Neolithisierungsregionen im östlichen Karpatenbecken, im Donauraum, in Slawonien und in Dalmatien gingen im Verlauf des 6. Jahrtausends v. u. Z. neue Kolonisationsprozesse aus, die spätestens um 5700 v. u. Z. unter anderem auch die Flusstäler der Mittelgebirgszone Zentralbosniens erfassten.

Von Beginn des Neolithikums an nahm Zentralbosnien in kultureller Hinsicht eine Mittelstellung zwischen neolithischen Gesellschaften Dalmatiens und dem Donaugebiet ein, die sich in hybriden Bezügen materieller Kultur manifestiert und auf die Bedeutung der Region in der großräumigen Kommunikation hinweist. ${ }^{1}$

Anknüpfend an eine eindrucksvolle Forschungsgeschichte, die im späten 19. Jh. mit den Grabungen in Butmir begann und deren Intensität während der jugoslawischen Ära einen Höhepunkt erlebte, fanden in Kooperation zwischen dem Bosnisch-Herzegowinischen Landesmuse-

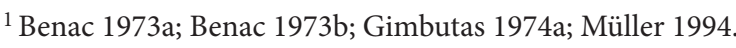

um Sarajewo (Zemaljski Muzej), dem Zentrum für Balkanforschung der Akademie der Wissenschaften und Künste Bosnien-Herzegowinas, der Römisch-Germanischen Kommission Frankfurt a. M. und der Christian-Albrechts-Universität zu Kiel umfangreiche archäologische Feldforschungen in der Region um die Städte Visoko und Kakanj statt. ${ }^{2}$ Das Ziel des Projektes „Rekonstruktion spätneolithischer Siedlungsprozesse in Zentralbosnien" bestand darin, exemplarisch für das südosteuropäische Spätneolithikum die ökonomische, soziale und demographische Entwicklungsdynamik in einer Siedlungskammer mit einem differenzierten Siedlungssystem mit Orten unterschiedlicher Größe zu rekonstruieren. Im Zentrum dieser Forschungen stand die Siedlung Okolište, die aufgrund ihrer Größe von fast 7 ha innerhalb Zentralbosniens singulär ist.

Ergänzend $\mathrm{zu}$ den Feldforschungen in Okolište fanden Prospektionen und Ausgrabungen an anderen, meist deutlich kleineren Siedlungsplätzen der Region Visoko und Kakanj statt, um die ökonomischen und sozialen Beziehungen zwischen Orten innerhalb der Mikroregion zu klären. Die archäologischen Untersuchungen erfolgten in Verbindung mit botanischen, zoologischen und geomorphologischen Untersuchungen, die sowohl Informationen zur Ökonomie neolithischer Gesellschaften als auch zur Landschaftsentwicklung in prähistorischer und historischer Zeit Zentralbosniens liefern. ${ }^{3}$

\footnotetext{
2 Zusammenfassend Hofmann u. a. 2006; 2008/09; Müller u. a. 2013 b.

${ }^{3}$ Benecke 2006; Dörfler 2006; Kučan 2006; Wolters / Bittmann 2006; Bultmann 2012; Benecke 2013; Dörfler 2013;
} 
Der vorliegende Beitrag stellt eine Zusammenfassung wichtiger Ergebnisse des Projektes dar, das unter Leitung von Zilka KujundžićVejzagić (Sarajewo), Johannes Müller (Kiel), Knut Rassmann (Frankfurt) stand. Der Autor war in die Forschungen im Rahmen seiner Dissertation involviert, in der er sich mit Hausarchitektur, Siedlungsstrukturen sowie der Entwicklung keramischer Fundgattungen im Neolithikum und frühen Äneolithikum (Gefäßkeramik, Figurinen u. a.) auseinandersetzte. ${ }^{4}$ Die Entwicklung dieser archäologischen Quellengattungen wird darin in zeitlicher Tiefe verfolgt, zur lokalen und regionalen Siedlungsdynamik in Beziehung gesetzt sowie sozialhistorische und sozioökonomische Implikationen diskutiert.

\section{Feldarbeiten in der Region Visoko und Kakanj}

Zwischen 2002 und 2008 erfolgten in der Region Visoko und Kakanj umfangreiche Feldforschungen. Mehrere Feldkampagnen konzentrierten sich auf den Fundplatz Okolište, der im Norden des Visokobeckens auf einer pleistozänen Schotterterrasse in unmittelbarer Nachbarschaft zur Niederung der Bosna liegt (Abb. 1-2). Die Siedlung wurde auf einer sanft nach Norden ansteigenden Geländezunge an der Schnittstelle zwischen für Ackerbau geeignetem Hinterland und der Flussaue der Bosna gegründet. Verglichen mit anderen Fundplätzen verfügte das Siedlungsumfeld über ein hohes agrarisches Potential, was die Wahl des Standortes beeinflusst und Entwicklung der Siedlung vermutlich begünstigte. ${ }^{5}$

Die Fundstelle bildet einen an der Peripherie verflachten, Nordost-Südwest ausgerichteten Siedlungshügel über einer ovalen, 270 x $265 \mathrm{~m}$ messenden Grundfläche. Ursprünglich besaß das Siedlungsareal eine Größe von 6,85 ha, von denen allerdings bis heute etwa 0,45 ha durch die Aktivität des Flusses Bosna verloren gegangen sind. Das Volumen der durch anthropogene Tätigkeit entstandenen Ablagerungen beträgt zwischen $110.000 \mathrm{~m}^{3}$ und $130.000 \mathrm{~m}^{3}$. Das Relief des Siedlungshügels lässt eine deutliche Terras-

Dreibrodt u. a. 2013a; Dreibrodt u. a. 2013b; Kroll 2013a; Kroll 2013b; Kroll in Vorbereitung.

${ }^{4}$ Hofmann 2013.

${ }^{5}$ Bultmann 2012. sierung erkennen, die mit variierenden Mächtigkeiten anthropogener Ablagerungen zwischen 1-3 m korreliert.

In nördlichen Hälfte der Siedlung wurden großflächige geomagnetische Prospektionen durchgeführt; im heute durch das Dorf Radinovići überbauten südlichen Teil war dies nur auf sehr kleinen Flächen möglich (Abb. 3). Im Bild der Geomagnetik können unterschiedliche Arten von Anomalien unterschieden werden: in Zeilen angeordnete verbrannte und unverbrannte Hausstrukturen, ein ausgedehntes mehrphasiges Grabensystem, geomorphologische Strukturen sowie moderne Störungen.

In der Siedlung Okolište erfolgten archäologische Ausgrabungen in den räumlich getrennten Grabungsflächen 1-9, die eine Fläche von ca. $1200 \mathrm{~m}^{2}$ und ein Aushubvolumen von etwa $1000 \mathrm{~m}^{3}$ umfassten (Abb. 3). Außerhalb des Siedlungsareals lag Grabungsfläche 10 (Schnitte 90 und 91) in einem verlandeten Arm der Bosna, die durch die geoarchäologische Arbeitsgruppe der Universität Kiel untersucht wurde und der Erforschung der holozänen Flussgeschichte der Bosna diente. ${ }^{6}$

Die Grabungen in den Flächen 1, 3 und 4 waren auf jeweils den obersten Meter der anthropogenen Ablagerungen fokussiert: Dieser Grabungsstrategie lag die Hypothese zugrunde, dass die im Plan der Geomagnetik sichtbaren Hausbefunde einer Siedlungsphase angehören, die bei einem großen Brandereignis zerstört wurden. Die Architektur und die Ausstattungsreste unterschiedlicher, räumlich klar getrennter Häuser bzw. Gruppen von Häusern sollten miteinander verglichen werden, um Aussagen zur ökonomischen und rituellen Bedeutung von Haushalten treffen zu können, die als wichtigste ökonomische und soziale Einheit in neolithischen Gesellschaften angesehen werden. Einen zweiten Schwerpunkt der Ausgrabungen bildete in den Flächen 2, 5, 6, 7 und 8 die Untersuchung peripherer Siedlungsbereiche und des Grabenwerkes, dessen komplexe Struktur eine längere Entwicklung erkennen ließ.

Im Umfeld der Siedlung Okolište wurden systematische Feldbegehungen durchgeführt. ${ }^{7}$ Ferner erfolgten an acht bereits bekannten Sied-

\footnotetext{
${ }^{6}$ Dreibrodt u. a. 2013a; Dreibrodt u. a. 2013b.

${ }^{7}$ Hofmann / Müller-Scheeßel 2013c.
} 


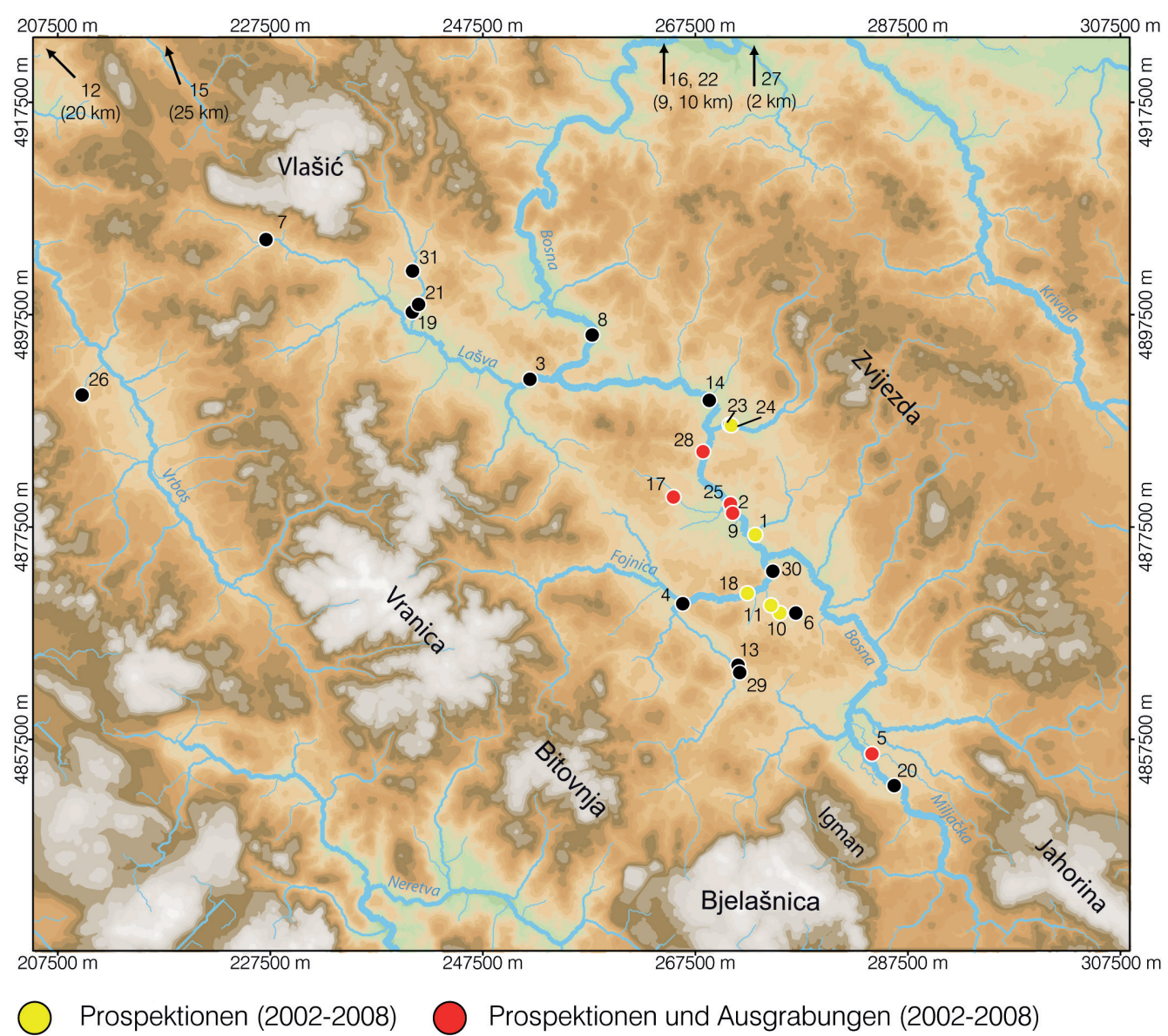

Abb. 1 Karte von Zentralbosnien mit Lage von neolithischen und frühäneolithischen Fundplätzen. 1 Arnautovići; 2 Batare; 3 Borak; 4 Brdo; 5 Butmir; 6 Čifluk; 7 Crkvine; 8 Drivuša; 9 Donje Moštre; 10 Dvor; 11 Ginje; 12 Gradina (Bocac); 13 Han Ploča; 14 Kakanj (Plandište); 15 Kovačica; 16 Kraljevina; 17 Kundruci; 18 Lopata; 19 Mujevine; 20 Naklo; 21 Nebo; 22 Novi Šeher; 23 Obre I (Raskršće); 24 Obre II (Gornje Polje); 25 Okolište; 26 Prusac (Biograd); 27 Tuk; 28 Zagrebnice; 29 Zagrebnjaca; 30 Zbilje (Krstac); 31 Gradina (Alihodža)

lungsplätzen geomagnetische Messungen, Geländeaufnahmen, Bohrungen und in zwei Fällen systematische Oberflächenabsammlungen. ${ }^{8} \mathrm{Ba}-$ sierend auf den Ergebnissen dieser Prospektionen wurden die Siedlungsplätze Donje Moštre, Kundruci und Zagrebnice für systematische Ausgrabungen ausgewählt. ${ }^{9}$

Den 3,5 ha großen Fundplatz Donje Moštre zeichnet seine Lage nur $900 \mathrm{~m}$ südlich von Okolište am linken Ufer des Flusses Bosna aus. Durch die Grabungen sollte hier unter anderem

\footnotetext{
${ }^{8}$ Ebenda. Arnautovići, Donje Moštre, Zagrebnice, Kundruci, Obre Gornje Polje, Dvor, Lopate, Okolište. Die Existenz einer Siedlung im Stadtgebiet von Visoko im Umfeld der Fojnica-Mündung in die Bosna konnte nicht bestätigt werden, obwohl wir sie weiterhin für wahrscheinlich ercheint halten.

${ }^{9}$ Furholt 2012; Furholt 2013; Hofmann / Müller-Scheeßel 2013a; Müller-Scheeßel / Hofmann 2013a.
}

geklärt werden, ob die beiden Siedlungen gleichzeitig sind oder unterschiedlichen Phasen der Urgeschichte angehören. Der nur 0,3 ha große Fundplatz Kundruci repräsentiert eine Gruppe von deutlich kleineren Siedlungen in höheren gelegenen Seitentälern westlich und südwestlich des Visokobeckens, die sich durch eine geschützte Lage auf Spornen auszeichnen. Neben chronologischen Fragen sollten mögliche Funktionsunterschiede zu Siedlungen im Haupttal der Bosna bzw. Spezialisierungen geklärt werden. Die 1 ha große Siedlung Zagrebnice zeichnet sich durch eine besonders verkehrsgünstige Lage in einem engen Durchbruchstal nördlich von Okolište aus, wo nur ein eingeschränktes agrarisches Potential zur Verfügung stand. 


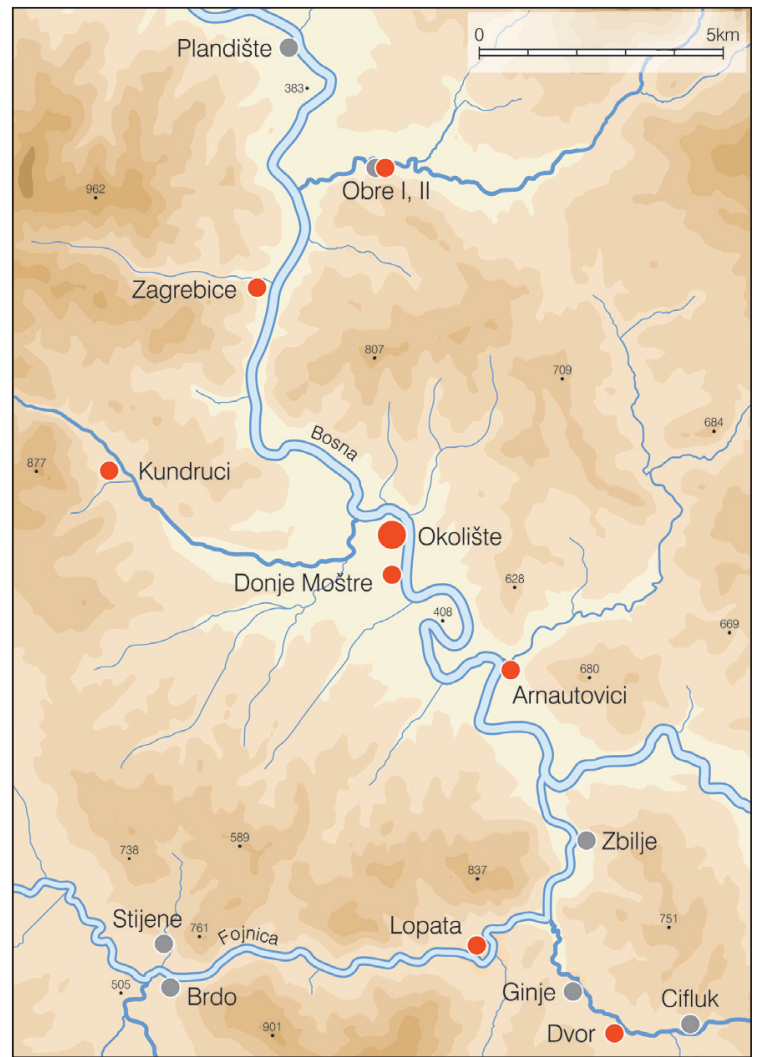

Abb. 2 Neolithische und frühäneolithische Fundplätze im Visokobecken, dem südlichen Teil des KakanjBeckens und dem Flusstal der Fojnica

\section{Chronologie von Okolište}

Ein Chronologiemodell von Okoliste, das basierend auf unterschiedlichen Beobachtungen und Methoden erarbeitet wurde, sieht drei Hauptphasen (1-3) und alternativ neun Phasen (1-9) vor (Abb. 4). ${ }^{10}$ Die Basis für diese Differenzierung bilden die dokumentierten Stratigraphien in den räumlich getrennten Grabungsflächen. Hinzu kommen Beobachtungen zum Relief des Siedlungshügels und variierende Mächtigkeiten der anthropogenen Ablagerungen. Zur absolutchronologischen Datierung stehen $29{ }^{14} \mathrm{C}$-Daten zur Verfügung. Unterstützend wurden außerdem Seriationen von Vergesellschaftungen morphologischer Merkmale und Verzierungen der Gefäßkeramik mittels Korrespondenzanalysen vorgenommen.

Von zentraler Bedeutung war die Feststellung, dass die in der Geomagnetik sichtbaren Anomalien nicht - wie anfangs angenommen

\footnotetext{
${ }^{10}$ Hofmann 2013, 54, Abb. 15 und 305, Abb. 185.
}

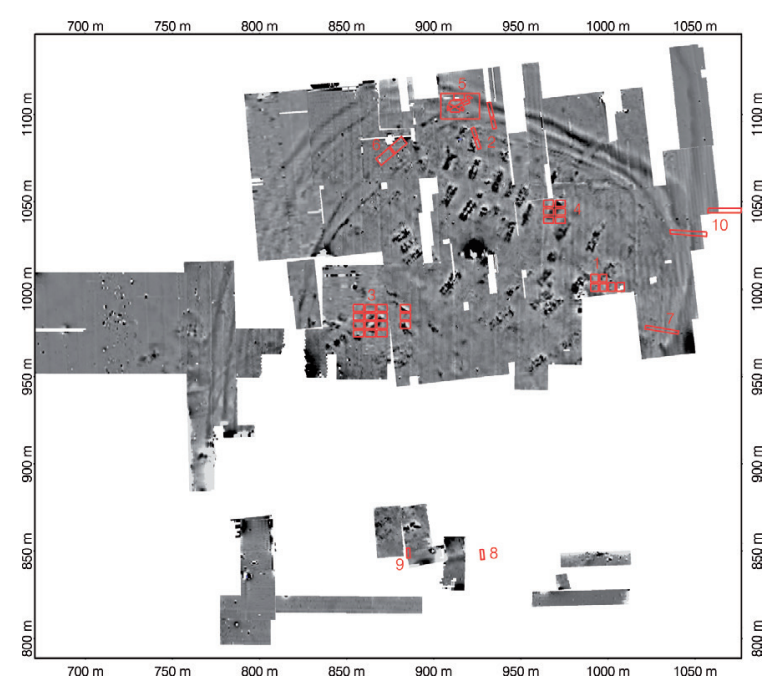

Abb. 3 Okolište, Plan der geomagnetischen Prospektion und Lage der Grabungsflächen

- in jedem Fall gleichzeitig sind, sondern teils unterschiedlichen Siedlungsphasen angehören. Die Unterscheidung der Hauptphasen 1 und 2 beruht auf der stratigraphischen Beobachtung, dass das Grabenwerk im Nordwesten der Siedlung verlegt und die Größe der Siedlung damit auf 5,6 ha verkleinert wurde. Alle Befunde aus der Zeit vor dieser Siedlungsverkleinerung gehören zu Hauptphase 1. Zu Hauptphase 3 werden die Ablagerungen einer deutlich erhöhten Terrasse im Nordosten bzw. Osten der Siedlung gerechnet. Erosion als Ursache für die Asymmetrie des Siedlungshügels wird ausgeschlossen. Vielmehr wird davon ausgegangen, dass die Terrassierung des Tells das Ergebnis einer erneuten Verkleinerung der Siedlung auf 1,2 ha darstellt.

Nach Bayesschen Modellierungen der ${ }^{14} \mathrm{C}$ Daten wurde die Siedlung mit der höchsten Wahrscheinlichkeit zwischen 5200 und $5150 \mathrm{v}$. u. Z. gegründet und um 4700 v. u. Z. endgültig aufgegeben. Die erste Siedlungsverkleinerung erfolgte um 5000 v. u. Z. und die zweite um 4850 v. u. Z.

Die Untergliederung in neun Siedlungsphasen beruht im Wesentlichen auf der Verknüpfung der unterschiedlichen Teilstratigraphien und der darin enthaltenen Schichtenformationen als höchster Ebene der Befundgruppierung. Befunde innerhalb der gleichen Fläche wurden dann unterschiedlichen Phasen zugerechnet, wenn Änderungen der Bebauungsstruktur oder der Bauweise von Häusern nachweisbar waren. Die 


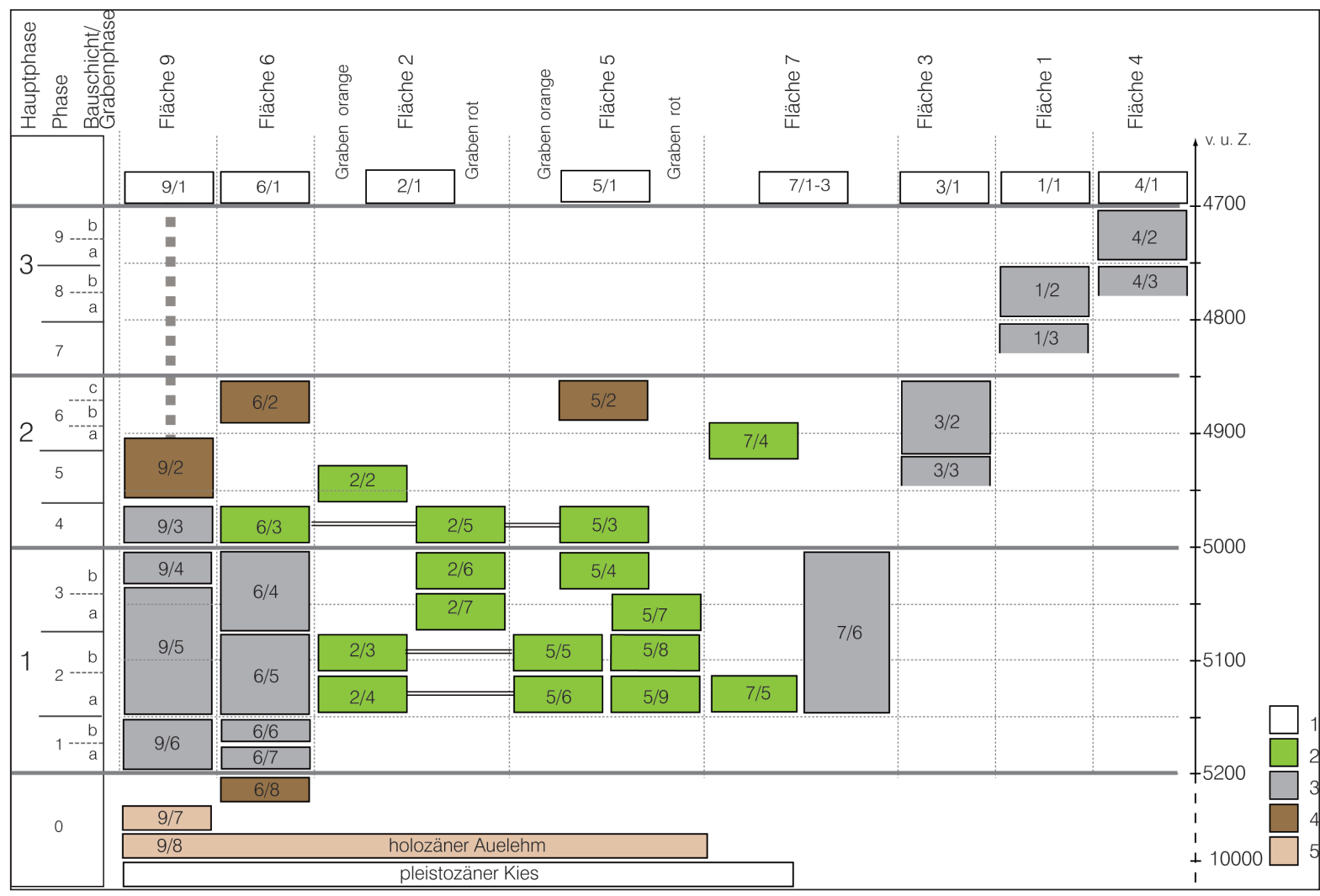

Abb. 4 Okolište, relativ- und absolutchronologische Gliederung der Schichtenformationen und ihre Zuordnung zu Hauptphasen, Siedlungsphasen und Bauschichten bzw. Grabenphasen nach Hofmann 2013, Abb. 185. 1 rezenter Ah-Horizont bzw. nachneolithisches Kolluvium; 2 Grabenverfüllung; 3 Siedlungsschicht; 4 begrabener Humus; 5 frühholozäner Auelehm

durchschnittliche Dauer dieser Siedlungsphasen beträgt 50 Jahre. In den zentralen Grabungsflächen ist in den Hausstellen eine noch feinere chronologische Untergliederung möglich, die sich auf die durchschnittliche Lebensdauer eines Hauses von 33 Jahren bezieht.

\section{Hausarchitektur in Okolište}

In den Grabungsflächen 1, 3, 4, 6 und 9 wurden die Reste von insgesamt 39 Häusern festgestellt, die teilweise oder vollständig innerhalb der ausgegrabenen Areale lagen. ${ }^{11}$ An der Basis des Siedlungshügels wurden in den Flächen 6 und 9 Grubenobjekte mit geraden Außenkanten und einer differenzierten Verfüllung aus zahlreichen dünnen, an Holzkohle und verkohlten botanischen Makroresten reichen Straten dokumentiert, die als Reste eingetiefter Bauten interpretiert werden (Häuser 38, 41, 42). Vollständig

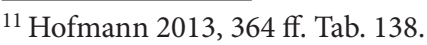

wurde Haus 38 in Schnitt 6 untersucht, das eine Größe von $8 \times 2 \mathrm{~m}$ besaß und bis zu $0,4 \mathrm{~m}$ in den anstehenden Boden eingetieft war. Neben der Dimension war vor allem die Ausrichtung des Objektes für die Interpretation als Haus ausschlaggebend. Zudem stammt aus der Siedlung Obre Gornje Polje ein ähnlicher Befund, an dem zusätzliche Details wie Türangelsteine und Reste der Dachdeckung erhalten waren. ${ }^{12}$

Bereits ab der zweiten Siedlungsphase wurden in Okolište ausschließlich rechteckige ebenerdige Häuser mit Grundflächen zwischen $30 \mathrm{~m}^{2}$ und $50 \mathrm{~m}^{2}$ errichtet. Detaillierte Rekonstruktionen dieser Häuser waren nur in Ausnahmefällen möglich (Abb. 5). ${ }^{13}$ In konstruktiver Hinsicht handelte es sich um Pfostenbauten mit Mittelpfostenreihen, deren Dächer auf Pfetten ruhten. Die Konstruktion der Wände entsprach jener, wie sie in Obre Gornje Polje dokumentiert wurde ${ }^{14}$.

\footnotetext{
12 Benac 1973 b, 30 ff.

${ }^{13}$ Vgl. Hofmann 2013, 368 Abb. 233-234.

${ }^{14}$ Benac 1973b.
} 


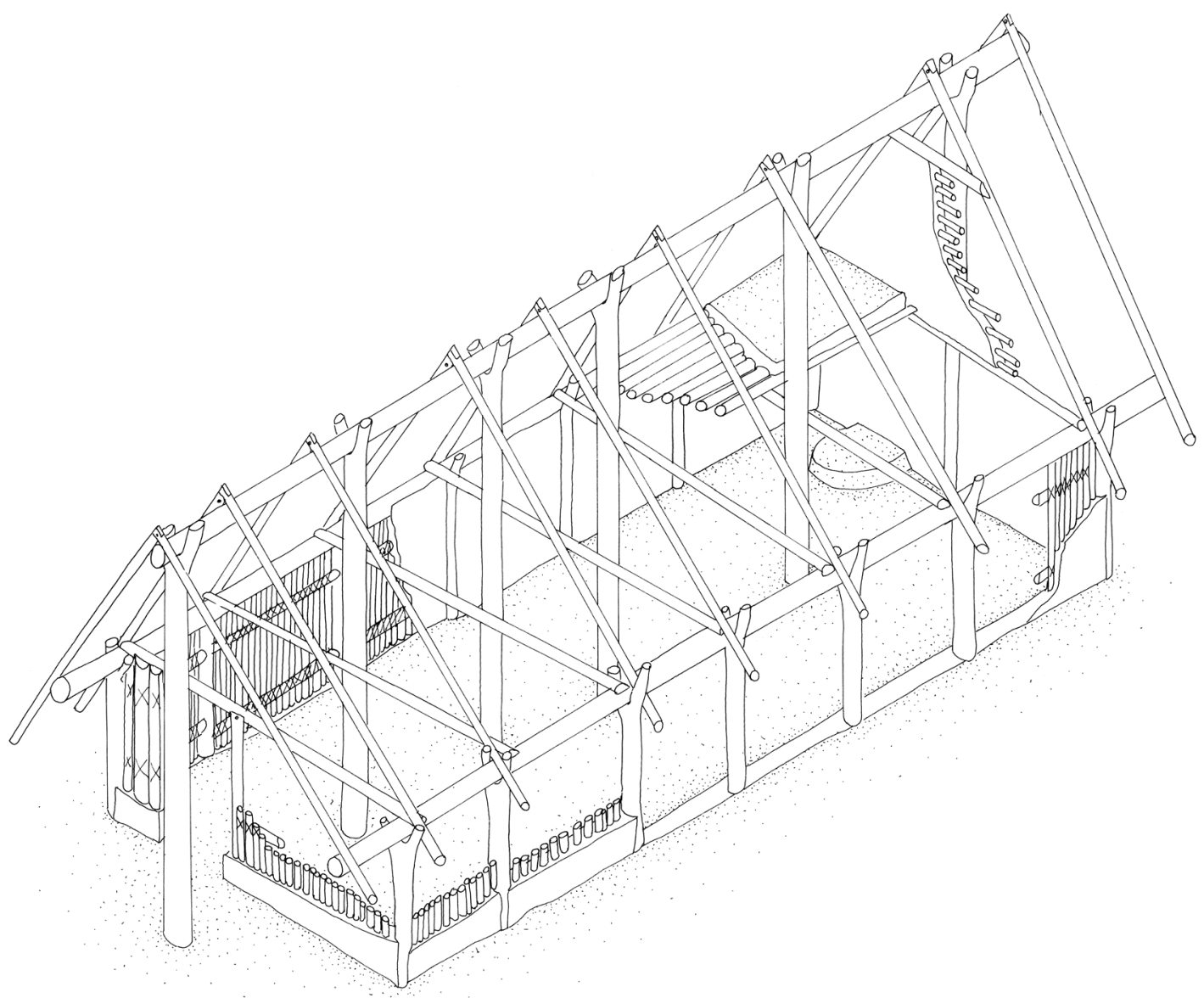

Abb. 5 Okolište, Rekonstruktion von Haus 1 in Grabungsfläche 3

In der letzten Siedlungsphase 9 ist eine deutliche Vergrößerung der Häuser auf ca. $80 \mathrm{~m}^{2}$ festzustellen. Vorzugsweise sind es diese vergrößerten Bauten, die in der geomagnetischen Prospektion im Nordosten der Siedlung sichtbar sind.

Mit durchschnittlich $4 \mathrm{~m}$ waren die Häuser in Okolište im Mittel $1 \mathrm{~m}$ schmaler als jene aus Obre Gornje Polje. ${ }^{15}$ Soweit Erkenntnisse zu konkreten Grundrissen vorliegen, handelt es sich um einräumige Bauten. Weder in Obre Gornje Polje noch in Okolište gibt es Belege für Häuser mit mehreren Feuerstellen, wie sie zum Beispiel für das zentrale Balkangebiet und Ostungarn charakteristisch sind.

Von den 39 in Okolište dokumentierten Häusern waren 13 teilweise oder vollständig verbrannt, während es sich bei 26 Gebäuden um Reste unverbrannter Bauten handelte. Die verbrannten Häuser kommen gehäuft in den jüngsten Sied-

\footnotetext{
${ }^{15}$ Hofmann u. a. 2006, $91 \mathrm{f}$.
}

lungsschichten vor. Allerdings muss vorläufig offen bleiben, ob dies auf der oben erläuterten Grabungsstrategie beruht, oder möglicherweise als Ausdruck verstärkter sozialer oder kriegerischer Konflikte gedeutet werden darf.

\section{Bebauungsstruktur in Okolište und Bevölkerungsgrößen}

Die Bebauungsstruktur in der Siedlung Okolište konnte am großflächigsten in Grabungsfläche 3 rekonstruiert werden. ${ }^{16}$ Hier gehörten neun vollständig oder teilweise freigelegte Hausstellen zu drei parallelen Häuserzeilen (Abb. 6 a). Zwischen den Langseiten der giebelständigen Häuser lagen 1-1,5 m schmale Gassen, die aufgrund des notwendigen Dachüberstandes nicht begehbar

\footnotetext{
${ }^{16}$ Hofmann u. a. 2006, 74 ff.; Hofmann u. a. 2008/09, 41 ff.; Hofmann 2013, $91 \mathrm{ff}$.
} 


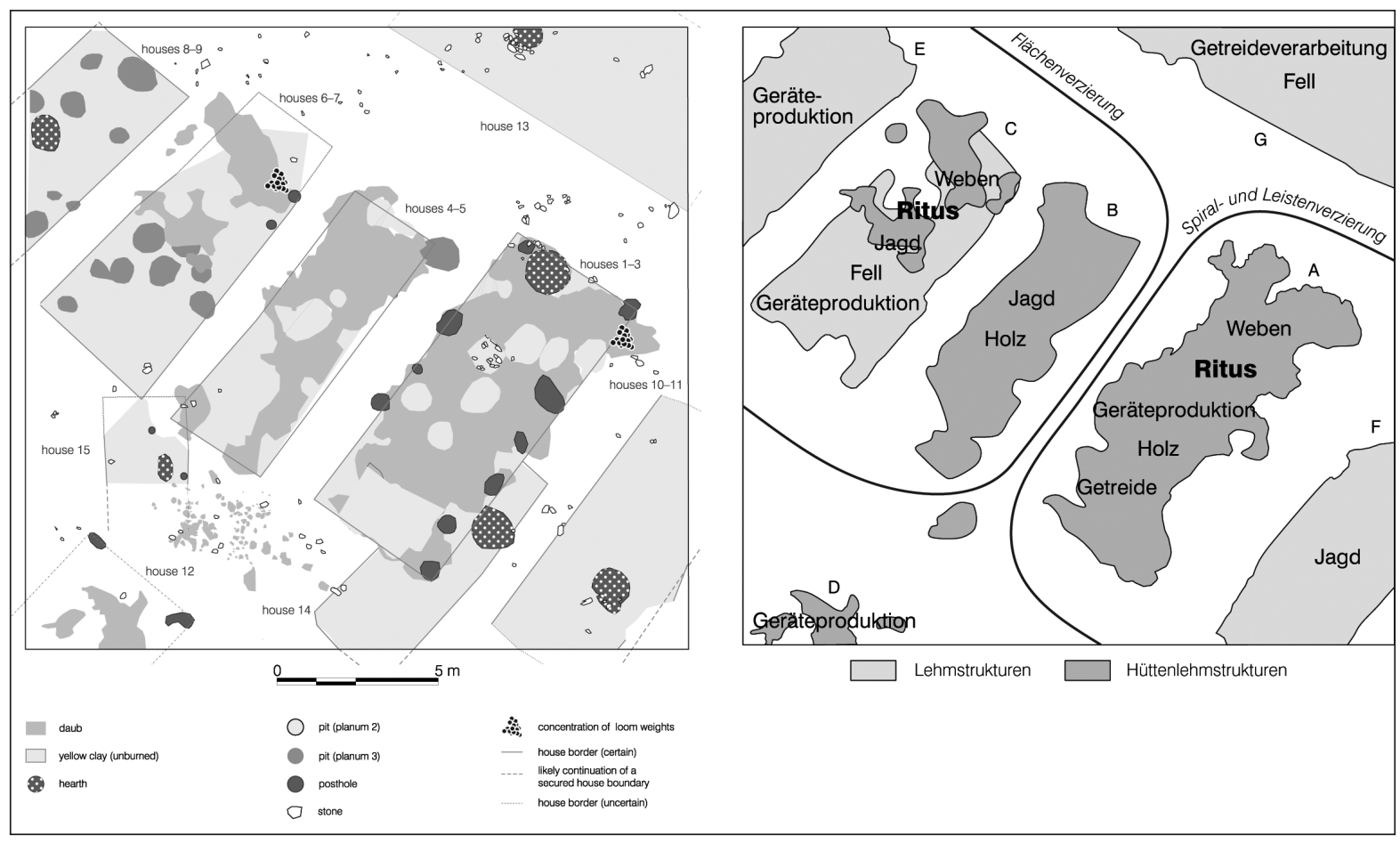

Abb. 6 Okolište, Grabungsfläche 3 a) Vereinfachter Befundplan nach Hofmann u. a. 2006, Abb. 27; b) nach Müller u. a. 2013b, Abb. 45

gewesen sein können. Zwischen den Zeilen bilden 5-6 $\mathrm{m}$ breite Wege die Erschließungsachsen.

Im Nordosten der Siedlung ist das Grabenwerk durch eine Toranlage unterbrochen (Abb. 3). Im Inneren der Siedlung ist diesem Tor eine Zone vorgelagert, die im Bild der geomagnetischen Prospektion fast keine Befunde zeigt und die deshalb als mögliche Hauptstraße interpretiert wurde, die die Siedlung in Nordost-Südwest-Richtung durchzog (Abb. 3). Indirekt wird dies durch Grabungen und ergänzende Bohrungen in Grabungsfläche 4 bestätigt, wo eine Bebauung nur in den jüngeren Siedlungsphasen nachgewiesen werden konnte.

An den einzelnen Hausstellen in Fläche 3 lagen jeweils die Reste von zwei oder drei Häusern übereinander. Die zugehörigen Häuser waren teils verbrannt und teils unverbrannt. Diese unterschiedlichen Erhaltungszustände werden als Ergebnis eines zeitlich gestaffelten Auflassungsprozesses des Siedlungsareals interpretiert, der sich über die Dauer von mindestens einer Hausgeneration hingezog. Diese Deutung wird auch durch ähnliche Befunde in anderen Siedlungsflächen untermauert (Flächen 1 und 4). In allen untersuchten Flächen erwies sich die
Bebauungsdichte als erheblich dichter, als es zunächst durch die geomagnetische Prospektion angezeigt war.

De grundlegende Struktur der Bebauung mit Nordost-Südwest orientierten Häusern in Nordwest-Südost verlaufenden Häuserzeilen wurden während der gesamten Siedlungsdauer von Okolište beibehalten. Allerdings erfolgten nach spätestens drei Hausgenerationen gewisse Verschiebungen der Hausreihen. Dabei fand eine zunehmende Verdichtung der Bebauung von anfangs $16 \%$ auf bis zu $55 \%$ bebauter Fläche in Phase 6 statt. Die wiederholten Erneuerungen der Bebauung führten zu einer sukzessiven Änderung der Ausrichtung von Häusern um insgesamt $20 \mathrm{Grad}$, wobei allerdings die Kriterien für die Ausrichtung der Häuser unklar blieben.

Bei der Errichtung und der Nutzung eines Hauses akkumulierten durchschnittlich 15-20 $\mathrm{m}^{3}$ Erdmaterial. Legt man das Gesamtvolumen anthropogener Ablagerungen zugrunde, wurden in Okolište insgesamt 5000-6000 Häuser errichtet (Tab. 1). ${ }^{17}$ Schlüsselt man diese Zahl auf

\footnotetext{
17 Die Methode, ausgehend von Volumina einzelner Häuser die Gesamtzahl von Häusern auf die gesamte Siedlung hochzurechnen, wurde erstmals im Jahr 2006 im Rahmen
} 


\begin{tabular}{|c|c|c|c|c|c|c|c|c|c|}
\hline 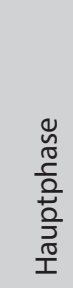 & 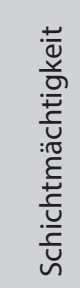 & 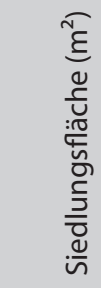 & 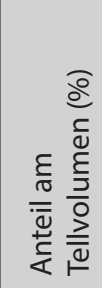 & 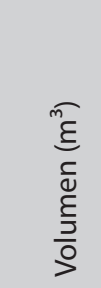 & 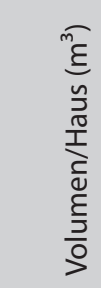 & 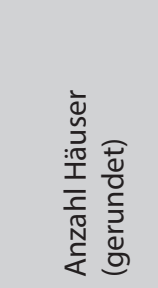 & 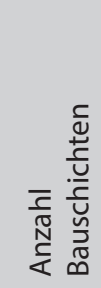 & 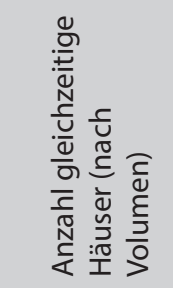 & 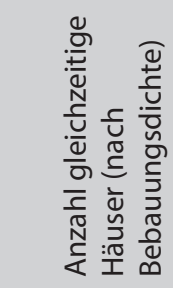 \\
\hline 1 & 0,8 & 68500 & 40 & 40000 & $15-20$ & $2000-2600$ & 4 & ca. $500-650$ & ca. $550 * 1$ \\
\hline 2 & 1,2 & 56000 & 50 & 50000 & 15 & 3300 & 6 & ca. 550 & ca. $750 * 2$ \\
\hline 3 & 1,0 & 12000 & 10 & 10000 & 20 & 500 & 5 & ca. 100 & ca. $123 * 3$ \\
\hline
\end{tabular}

Tab. 1 Okolište, Hochrechnung der Anzahl gleichzeitiger Häuser für die Hauptphasen 1-3 basierend auf dem Tellvolumen, der Siedlungsfläche, der Anzahl von Bauschichten, des ermittelten Volumens einzelner Häuser und der Bebauungsdichte nach Hofmann 2013, Tab. 146. ${ }^{*}$ Gerechnet wurde mit mittlerem Bebauungsanteil von $30 \%$ und einer mittleren Hausgröße von $36,9 \mathrm{~m}^{2}$, die allerdings nur auf zwei Belegen beruhen, die zudem sehr unterschiedlich sind. ${ }^{\star} 2$ Grabenbereiche müssten noch abgezogen werden. ${ }^{*} 3$ Gerechnet wurde mit einem Bebauungsanteil von $55 \%$ entsprechend den Gegebenheiten in Schichtenformation Oko 3/2

\begin{tabular}{|l|l|l|l|l|l|l|l|l|}
\hline $\begin{array}{l}\text { Siedlun- } \\
\text { gsperiode }\end{array}$ & $\begin{array}{l}\text { Siedlungs- } \\
\text { fläche (ha) })\end{array}$ & $\begin{array}{l}\text { bebaute } \\
\text { Fläche } \\
(\%)\end{array}$ & $\begin{array}{l}\text { durchschnittliche } \\
\text { Hausgröße }\left(\mathrm{m}^{2}\right)\end{array}$ & $\begin{array}{l}\text { Anzahl } \\
\text { Häuser } \\
\text { (gerundet) }\end{array}$ & $\begin{array}{l}\text { Bewohner } \\
\text { /Haus }\end{array}$ & Dauer & Einwohner & $\begin{array}{l}\text { Bevölkerungs } \\
\text { wachstum/Jahr } \\
(\%)\end{array}$ \\
\hline $\begin{array}{l}\text { Starčeco- } \\
\text { Impresso }\end{array}$ & 1,8 & $30^{*}$ & $37^{*}$ & 146 & 5 & 200 & 730 & 0,25 \\
\hline Kakanj & 3 & 30 & $37^{*}$ & 240 & 5 & 300 & 1200 & 0,48 \\
\hline Butmir 1a/b & 12,5 & 30 & 37 & 1014 & 5 & 200 & 5070 & 0,13 \\
\hline Butmir 2a & 9,5 & 55 & 40 & 1306 & 5 & 150 & 6530 & 0,05 \\
\hline Butmir 2b & 9,5 & 55 & 40 & & 5 & 100 & & \\
\hline Butmir 3a & 9,5 & 63 & 80 & 750 & 10 & 150 & 7481 & $-0,63$ \\
\hline Butmir 3b & 3,7 & $63^{*}$ & 80 & 290 & 10 & 100 & 2900 &. \\
\hline Butmir 4 & 3,6 & - & $>100$ & & & 300 & &. \\
\hline
\end{tabular}

Tab. 2 Hochrechnung der für Okolište rekonstruierten Bevölkerungszahlen auf neolithische Siedlungen in Zentralbosnien nach Hofmann 2013, Tab. 171. Zugrunde liegen die Gesamtfläche von Siedlungen, die bebaute Fläche innerhalb der Siedlungen, durchschnittliche Hausgrößen und geschätzte Bewohnerzahlen. Mit * bezeichnete Maße wurden von zeitlich „angrenzenden" Siedlungsperioden " ̈̈bernommen. Das Bevölkerungswachstum wurde nach Herrmann u. a. 1990, 312 mit der Formel $r=\left(\sqrt[t]{P_{t} / P_{0}}-1\right) * 100$ berechnet, wobei $r$ die Wachstumsrate, $t$ die Zeitdauer, P0 die Bevölkerungsgröße vor Zeitdauer t und Pt die Bevölkerungsgröße nach Zeitdauer t bezeichnen

die dokumentierten Bauschichten und Schichtmächtigkeiten auf, dürften in Okolište in den Hauptphasen 1 und 2 ca. 500-600 Häuser gleichzeitig bestanden haben. Zu sehr ähnlichen Ergebnissen gelangt man ausgehend von den durchschnittlichen Hausgrößen und den ermittelten Bebauungsdichten. Bei einer durchschnittlichen

eines Vortrages bei der Tagung "Leben auf dem Tell als soziale Praxis" von K. Rassmann und J. Müller vorgestellt und später vom Verfasser erweitert.
Bewohnerzahl eines Hauses von fünf Personen würde dies im Fall von Okolište für eine Population von 2000-3 000 Einwohnern sprechen. Mit der Siedlungsverkleinerung um 4850 v. u. Z. ging die Bevölkerungsgröße dann deutlich auf etwa 500 Menschen zurück.

Andere neolithische Siedlungen Zentralbosniens wiesen grundsätzlich eine sehr ähnliche Bebauungsstruktur und Bebauungsdichte wie in Okolište auf, soweit Informationen aus Prospek- 
tionen und Grabungen vorliegen. Deshalb halten wir es für gerechtfertigt, die bei den Grabungen in Okolište festgestellte Häuseranzahl als Ausgangspunkt für Hochrechnungen regionaler Bevölkerungsgrößen und jährlicher Bevölkerungswachstumsraten $\mathrm{zu}$ nutzen (Tab. 2). Sind entsprechende Hochrechnungen auch mit zahlreichen Unsicherheiten und Fehlerquellen behaftet, können sie doch einen ungefähren Eindruck von der Größenordnung menschlicher Gesellschaften im Neolithikum und frühen Äneolithikum und Entwicklungstrends geben.

\section{Grabenwerk in Okolište}

Mit Ausnahme eines fraglichen Befundes in Arnautovići wurde in Zentralbosnien trotz umfangreicher Prospektionen und Ausgrabungen bisher in keiner anderen Siedlung als Okolište ein Grabenwerk nachgewiesen. ${ }^{18}$ Wir dürfen deshalb vorläufig davon ausgehen, dass das Grabenwerk ein Alleinstellungsmerkmal der mit Abstand größten Siedlung in der Region darstellt.

In dem Grabenwerk gruppieren sich jeweils mehrere Gräben zu breiten Grabensträngen mit vier bis fünf parallelen Gräben, deren Lage im Osten und Nordosten lange Zeit konstant blieb, im Westen und Nordwesten hingegen mindestens einmal an eine veränderte Siedlungsgröße angepasst wurde (Abb. 3). Im Süden des Dorfes gab es wahrscheinlich keine Gräben, da sich hier an eine Schleife der Bosna anschloss.

Die Gräben der Siedlung sind durch wiederholte Verfüllungen und Erneuerungen gekennzeichnet. Unterschiedliche Zusammensetzung von Verfüllschichten und variierende Funddichten belegen, dass Verfüllungen der Gräben in mehreren Schritten erfolgten, wobei die Gräben wiederholt zur Abfallentsorgung genutzt wurden. Im Verlauf eines solchen mehrphasigen Verfüllungsprozesses gelangte im Norden der Siedlung eine größere Anzahl menschlicher Knochen in die Verfüllung eines der Gräben. ${ }^{19}$ Die räumliche Verteilung, Anthropologie, Datierung und Erhaltungszustände der Knochen erscheinen am ehesten durch eine Seuche erklärbar.

Innerhalb der untersuchten Grabenstränge besaß der jeweils äußere Graben die tiefte Sohle.

\footnotetext{
${ }^{18}$ Hofmann / Müller-Scheeßel 2013c.

${ }^{19}$ Müller-Scheeßel u. a. 2007, 2009.
}

Bei der Diskussion, ob innerhalb eines Grabenstranges gleichzeitig mehrere Gräben existierten und in welchem Rhythmus die Gräben erneuert wurden, führte die Abwägung unterschiedlicher Argumente zu der Hypothese, dass in Hauptphase 1 zeitweise mehrere parallele Gräben bestanden, in Hauptphase 2 jedoch jeweils nur noch ein Graben existierte. In Hauptphase 3 war das Grabenwerk offenbar bereits aufgegeben worden. Entsprechend diesem Modell müssten in den Phasen 1-6 durchschnittlich alle 40 Jahre Grabenerneuerungen stattgefunden haben. Alternativ müsste man von einer deutlich häufigeren Erneuerung des Grabensystems ausgehen.

\section{Taphonomie, Abfallverhalten und Aktivitätszonen in Okolište}

Räumliche Verteilungen von Artefakten in Siedlungskontexten können nicht direkt funktional gedeutet werden, da sie in der Regel Ergebnis komplexer Depositionsprozesse sind. Die Identifizierung von Aktivitätszonen und auch die Verwendung der Inventare für chronologische Auswertungen erfordert deshalb eine quellenkritische Bewertung von Befunden und Fundinventaren. $\mathrm{Zu}$ diesem Zweck wurden in Okolište räumliche Verteilungen von Silex- und Keramikmengen (Anzahl, Gewicht, Typen) in Relation zu Aushubvolumina und die Fragmentierung (durchschnittliches Scherbengewicht) in unterschiedlichen Tiefen und Befundkategorien untersucht. $^{20}$

In den Freiflächen im Umfeld der freigelegten Häuser stammt die mit Abstand größte Menge an Gefäßkeramik und Silex in Okolište. ${ }^{21}$ Systematische Unterschiede hinsichtlich der Zusammensetzung von Keramikinventaren inner- und außerhalb der Häuser belegen, dass Hausabfall zumeist im unmittelbaren Umfeld der Häuser entsorgt wurde. Peripher gelegene Grabenbereiche wurden dagegen nur in geringerem Umfang zur Abfallentsorgung genutzt. Insgesamt deutet die Konzentration von Hausabfall im Umfeld der Häuser auf ein wenig nachhaltiges Abfallverhalten der Bewohner von Okolište hin. In einem dicht bebauten Dorf wie in Okolište dürfte dieses

\footnotetext{
${ }^{20}$ Müller-Scheeßel u. a. 2010c; Hofmann 2013, 229 ff.; Müller-Scheeßel 2013, $273 \mathrm{ff}$.

${ }^{21}$ Hofmann u. a. 2006; Hofmann u. a. 2008/2009.
} 
Verhalten zu erheblichen Belastungen der Menschen und der Einschränkung ihrer Lebensqualität geführt haben.

Die Fundinventare im Umfeld der Häuser stellen komplexe Gemenge unterschiedlicher taphonomischer Zustände dar: Nur ein relativ kleiner Teil der Keramik von ca. 15\% wurde noch im Kontext ihrer Verwendung (Biozönose) innerhalb der Häuser vorgefunden, während 57\% aus unterschiedlichen Abfallkontexten (Thanatozönose) und $28 \%$ aus noch stärker gestörten Kontexten (Taphozönose) stammen. Nur in einem Fall (Haus 1) wurde ein größeres Inventar eines abgebrannten Hauses in situ vorgefunden. In allen anderen Fällen waren die keramischen Hausinventare lediglich in Resten erhalten, da sie ausgeräumt oder erodiert waren. Eine gesonderte Kategorie stellen Hausstellen dar, die nach ihrer Auflassung als Abfallbereiche nachgenutzt wurden, wodurch es zu einer Vermischung unterschiedlicher Inventare kam.

Die räumlichen Verteilungen einiger Objektkategorien sind nachweislich nicht durch Abfallentsorgungsprozesse beeinflusst, sondern repräsentieren inner- und außerhäusliche Abfallsentsorgungsprozesse (Aktivitätszonen). ${ }^{22}$ Das Innere der Häuser diente zum Weben, zur Ausbewahrung von Vorräten, für rituelle Zwecke und zur Konsumption von Nahrung. Im Umfeld der Häuser wurden Silexartefakte hergestellt und mit Silex- und Felsgesteinartefakten gearbeitet (z. B. Holzverabeitung, Fellbearbeitung).

\section{Keramik als historische Quelle zum zentralbosnischen Neolithikum}

In einer Datenbank, die mittlerweile als InternetRessource frei zur Verfügung steht, sind neben Daten zu Befunden und Datierungen qualitative und quantitative Informationen zu sämtlichen Funden erfasst, die während der Ausgrabungen geborgen wurden. ${ }^{23}$ Die Funde werden im Heimatmuseum Visoko (bis einschließlich 2005) und im Bosnisch-Herzegowinischen Landesmuseum Sarajewo (ab 2006) aufbewahrt.

Eine der maßgeblichen Quellen für die Rekonstruktion der ökonomischen und sozialen Bezie-

\footnotetext{
${ }^{22}$ Hofmann u. a. 2006, 2008/09.

${ }^{23}$ Müller-Scheeßel / Hofmann 2013b; Müller-Scheeßel u. a. 2013.
}

hungen in den neolithischen Gesellschaften Zentralbosniens stellen Reste von Gefäßkeramik dar, die mit abgestufter Detailliertheit in mehreren Stichproben erfasst wurde: ${ }^{24}$ Einerseits wurden in einer sogenannten „Grobaufnahme“ sämtliche Artefakte nach Anzahl, Gewicht und Zugehörigkeit zu technologischen Gruppen (Waren) aufgenommen und im Hinblick auf Gefäßformen und Verzierungen grob klassifiziert. Andererseits wurden im Rahmen der sogenannten „Feinaufnahme“ von einer kleineren Stichprobe von 3200 Gefäßeinheiten detaillierte Informationen $\mathrm{zu}$ Technologie, Morphologie und Verzierungen erfasst. Abgesehen von der Gefäßkeramik wurden auch andere Objektkategorien aus Keramik - wie zum Beispiel anthropomorphe und zoomorphe Figurinen, Webgewichte und Spinnwirtel - beschrieben, systematisiert und als Quellen für soziale und ökonomische Fragen analysiert. ${ }^{25}$

Während der etwa 500 Jahre währenden Siedlungsdauer von Okolište unterlag die Gefäßkeramik einem starken technologischen und stilistischen Wandel. Die Keramik der frühesten Phase 1 von Okolište weist noch mehrheitlich Merkmale spätkakanj-zeitlicher Keramik auf. Charakteristisch sind unter anderem sehr dünnwandige, linear rot auf grau bemalte $\mathrm{Ge}$ fäße, zahlreiche plastische Applikationen und selten auch Barbotine-Verzierungen an grobkeramischer Ware.

In den Phasen 2-3 ist die Herausbildung von Butmir-Keramik zu beobachten. Innerhalb von etwa 100 Jahren setzen sich nach und nach die Magerung mittels gebrochenen Kalkstein, dunkel (reduzierend) gebrannte, teils polierte Waren, neue Gefäßformen sowie eingeritzte und eingestochene Flächenverzierungen durch. Wichtig für die Interpretation dieser Veränderungen ist, dass die Neuerungen in unterschiedlichen Herkunftsregionen lokalisiert werden können: dem ostadriatischen Raum (z. B. Kalkmagerung) und dem zentralen Balkangebiet (z. B. dunkel gebrannte Waren). Insgesamt deuten der Verlauf und die räumlichen Bezüge darauf hin, dass der beschriebene Wandlungsprozess maßgeblich auf einer Intensivierung überregionaler Kommunikationsbeziehungen beruht. Mit einiger Wahr-

\footnotetext{
${ }^{24}$ Hofmann 2013, $57 \mathrm{ff}$.

${ }^{25}$ Hofmann 2013, 221 ff., 357 ff., 361 ff.; Hofmann / Hofmann 2013.
} 
scheinlichkeit können hingegen größere Bevölkerungsbewegungen als Auslöser dieser Veränderungen ausgeschlossen werden (siehe unten).

Mittels zweier Quotienten kann die Geschwindigkeit des stilistischen Wandels der Gefäßkeramik quantifiziert werden. ${ }^{26}$ Dabei erweist sich die Periode zwischen 5200 und 5000 v. u. Z. als eine Phase forcierten Wandels, wohingegen nach 5000 v. u. Z. die Innovationsgeschwindigkeit mehr und mehr zurückgeht.

Als weitere übergeordnete Parameter wurden unter anderem die Häufigkeit von Warengruppen, die Diversität des Verzierungs- und Gefäßformenspektrums sowie die Verzierungsrate der Keramik untersucht. Letztere Parameter können unter den vermuteten Bedingungen einer Haushaltsproduktion als Indikatoren für soziale Komplexität interpretiert werden. Repräsentative Funktionen der Keramik in Bewirtungsritualen von miteinander in Wettbewerb stehenden Haushalten dürften dieser Korrelation zugrunde zu liegen.

Während die Diversität des Keramikspektrums bis Phase 4 zunimmt und dann konstant bleibt, ist die höchste Verzierungsrate in Phase 5 erreicht und ab Phase 8 wieder rückläufig. Demnach wäre für die Hauptphase 2 (Phasen 4-6) von der höchsten sozialen Komplexität in Okolište auszugehen. Insbesondere Inventare der Phasen 4-8 sind durch hohe Anteile polierter Feinwaren gekennzeichnet, die insbesondere als Tischgeschirr identifiziert werden können.

Erneut ein markanter Wandel ereignete sich in den letzten Phasen 8-9 von Okolište in Form einer stärkeren stilistischen „Internationalisierung" bzw. einer deutlichen Zunahme überregionaler Kommunikation in das Ostadriagebiet einerseits (Hvar-Lisičići) und das zentrale Balkangebiet und das östliche Karpatenbecken andererseits (Vinča, Theiss). Dieser Wandel ist verbunden mit dem Auftauchen neuer Gefäßformen, einer geringeren Häufigkeit von Feinware, einer generell größeren Keramikmenge, einer geringeren Verzierungsrate und mit einer gewissen Flüchtigkeit bei der Ausführung von Verzierungen. Die Häufigkeit von Indikatoren sozialer Komplexität ist in dieser Phase eindeutig rückläufig.

\footnotetext{
${ }^{26}$ Hofmann 2013, 356 f.
}

Anhand stilistischer und technologischer Merkmale lassen sich in Okolište klare Gefäßkategorien unterscheiden und bestimmten Funktionen zuweisen. Zum Beispiel weisen aus grober Keramik hergestellte Töpfe regelmäßig sekundäre Verfärbungen auf, die wahrscheinlich bei ihrer Verwendung bei thermischen Prozessen entstanden sind. Dagegen kann die Verwendung der meisten Schüsseln und Fußschalen bei thermischen Prozessen ausgeschlossen werden, da sie reduzierend gebrannt sind und sehr sorgfältige Oberflächenbehandlungen aufweisen. Diese Gefäßklassen dienten wahrscheinlich als Ess- und Trinkgeschirr.

Untersuchungen von Gefäßvolumina zeigen, dass die Gefäße generell zu klein waren, um für eine Langzeitlagerung größerer Vorratsmengen infrage zu kommen. Während der Besiedlungsdauer von Okolište sind auch keine markanten Volumenänderungen von Gefäßen nachweisbar, die andernorts auf Änderungen der Vorratskapazitäten hinzudeuten scheinen. Tischgeschirr repräsentiert etwa drei Viertel des Keramikbestandes in Okolište, während Gefäße zur Langzeitlagerung und Zubereitung von Nahrung vergleichsweise selten sind. Kartierungen belegen teils markante räumliche Verteilungsmuster von Gefäßklassen, die allerdings in den meisten Fällen eher durch Abfallentsorgungsprozesse zustande kommen und nur in Ausnahmefällen als Ergebnis von funktionalen Aktivitätsmustern interpretiert werden können.

\section{Chronologie regional - Siedlungsgeschichte der spätneolithischen Butmirgruppe}

Die Einbeziehung der regionalen Siedlungsdynamik in Zentralbosnien trägt entscheidend zum Verständnis der beschriebenen lokalen Entwicklung in Okolište bei. Grundlage für die Synchronisierung sind naturwissenschaftliche Datierungen und Untersuchungen zu Stratigraphie, Typochronologie und Sedimentationsraten aus Okolište, Kundruci, Zagrebnice, Donje Moštre und Butmir. In einem regionalen Chronologiemodell wurde versucht, die lokalen Sequenzen von Okolište und anderen Siedlungen mit den Chronologien früherer Forscher in Ein- 


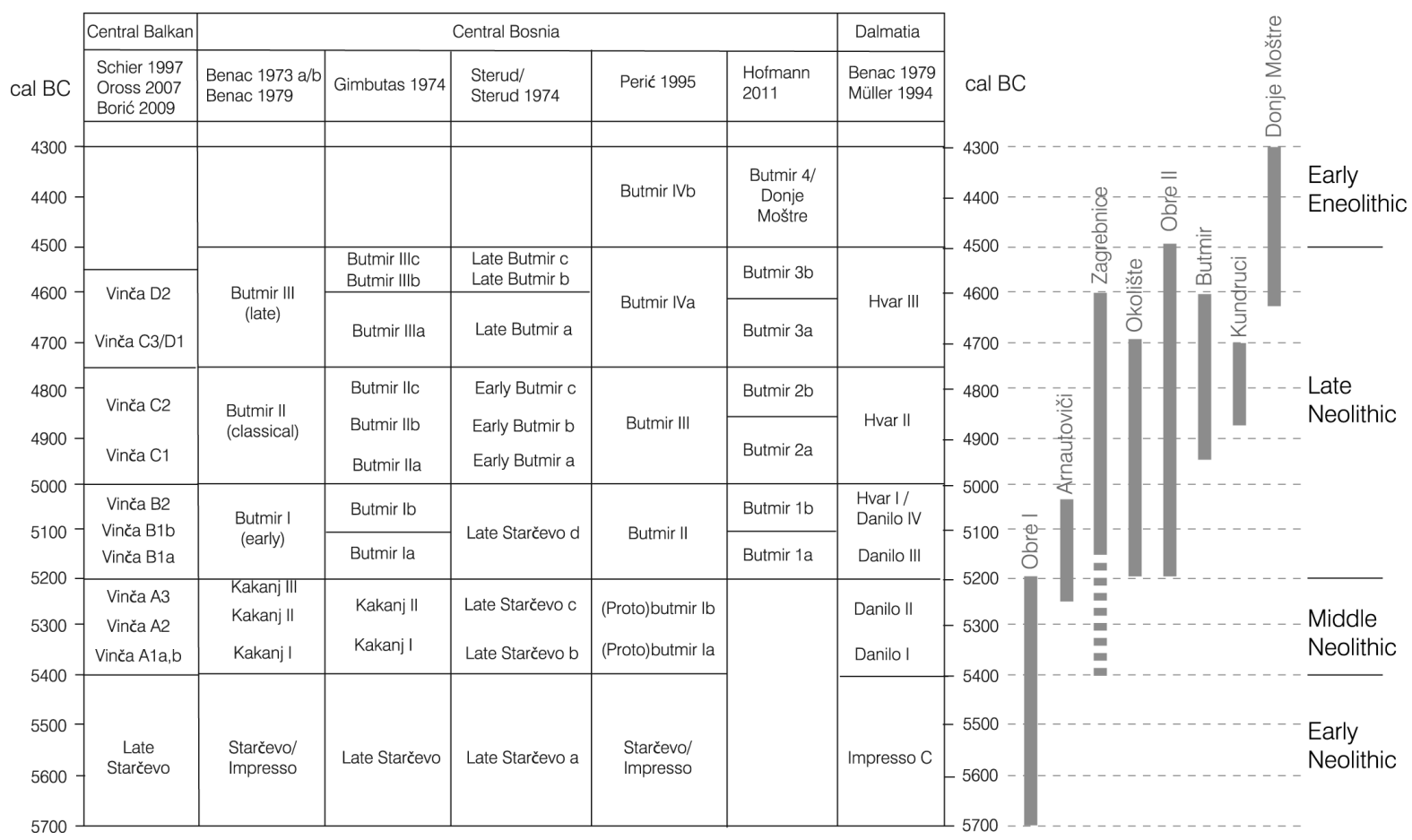

Abb. 7 Vergleichende Darstellung regionaler Chronologie systeme des Neolithikums und frühen Äneolithikums für Zentralbosnien und die Laufzeiten einiger Siedlungen

klang zu bringen. Dafür war insbesondere eine Synchronisierung mit den Sequenzen aus Obre Raskršće und Obre Gornje Polje herzustellen, die das „Rückgrat“ der bisherigen Periodisierungen darstellen.

Obwohl hinsichtlich der Synchronisierung der Siedlungen Obre Gornje Polje und Okolište derzeit noch Widersprüche zwischen typochronologischer Bewertung einerseits und naturwissenschaftlicher Datierung andererseits bestehen, wird für die spätneolithische Butmir-Gruppe eine Periodisierung vorgeschlagen, die sich in Grundzügen an jener von Alois Benac ${ }^{27}$ orientiert, jedoch auch die von Slaviša Perić ${ }^{28}$ eingeführte Spätphase einschließt (Abb. 7). Dieser Periodisierungsvorschlag versucht, einschneidende Entwicklungen der Siedlungsgeschichte zu berücksichtigen.

Nach derzeitiger Kenntnis bildet die Siedlungskammer um Kakanj und vermutlich das nördlich angrenzende Tal der Bosna zwischen 5800 und 5500 v. u. Z. die Kernregion der neolithischen Besiedlung Zentralbosniens. Erst etwas später wird das südlich angrenzende Viso-

\footnotetext{
${ }^{27}$ Benac 1973a; Benac 1993; Benac 1979.

${ }^{28}$ Perić 1995.
}

kobecken erschlossen, wo um 5200 v. u. Z. die Großsiedlung Okolište gegründet wurde. Wichtig erscheint die Erkenntnis, dass die Siedlung zu ihrer Gründungszeit kaum als Zentrum eines ansonsten durch kleine „Satelliten“-Siedlungen geprägten Siedlungssystems angesehen werden kann, sondern abgesehen von Arnautovići und vielleicht Zagrebnice offenbar die einzige Siedlung darstellte.

Um 5000 v. u. Z. wird mit Beginn der Periode Butmir 2 anhand von Siedlungsgründungen bei Sarajewo (Butmir) und Travnik (Nebo) eine substanzielle Ausweitung des neolithischen Siedlungsgebietes sichtbar. Etwa in der gleichen Zeit oder etwas früher wurde die Siedlung Arnautovići endgültig aufgegeben. In Okolište erfolgte die erste substanzielle Siedlungsverkleinerung, die allerdings mit einer zunehmenden Verdichtung der Bebauung einherging und deshalb wohl nicht mit einem Bevölkerungsrückgang zusammenhing.

Für Okolište erheblich einschneidender war die Verkleinerung der Siedlungsfläche auf nur noch 1,2 ha um 4850 v. u. Z., im Rahmen derer die Anzahl der Häuser auf ca. 100 zurückging. Spätestens seit dieser Zeit wurden in der höher gelegenen Region westlich und südwestlich des 


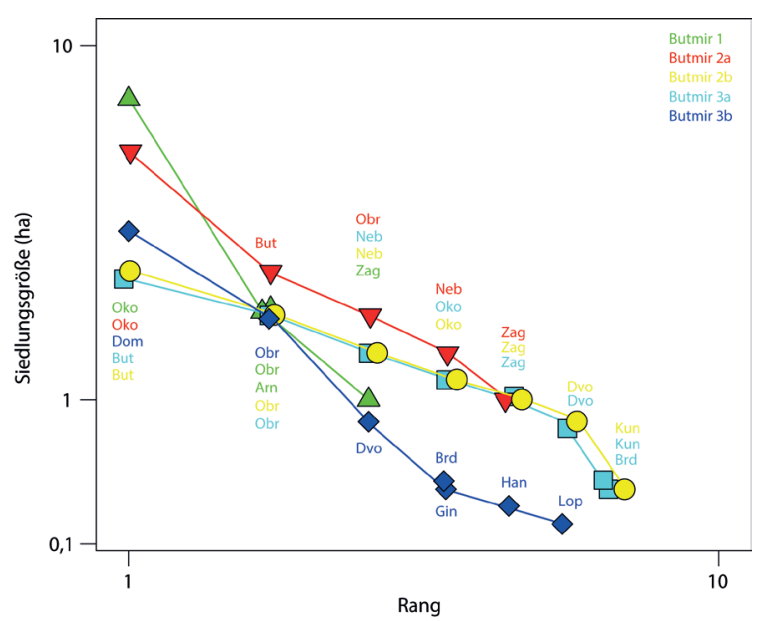

Abb. 8 Rang-Größen-Verteilungen spätneolithischer Siedlungen Zentralbosniens zwischen 5200 und 4500 v. u. Z. (Perioden Butmir 1-Butmir 3b).

Datengrundlage Hofmann 2013, Tabelle 170. Oko: Okolište, Dom: Donje Moštre, But: Butmir, Obr: Obre Gornje Polje, Arn: Arnautovići, Neb: Nebo, Zag: Zagrebnice, Dvo: Dvor, Brd: Brdo, Gin: Ginje, Han: Han Ploča, Lop: Lopate, Kun: Kundruci

Visokobeckens zahlreiche, mehrheitlich sehr kleine Siedlungen neu gegründet, die häufig auf geschützten Spornen liegen. Insgesamt fand also eine deutliche Nivellierung der zeitweise extremen Größenunterschiede von Dörfern statt. In Termini von Rang-Größen-Verteilungen (ranksize distribution) gesprochen fand eine Entwicklung von einer stark ,primaten ${ }^{c}$ zu einer tendenziell ,konvexen' Verteilung statt (Abb. 8). Später, am Übergang zum frühen Äneolithikum, bildeten sich wieder stärkere Unterschiede im regionalen Siedlungssystem hinsichtlich Größe und auch der Einwohnerzahl von Orten heraus, die als Dichothomie zwischen größeren Dörfern in den Haupttälern und deutlich kleineren Siedlungen in höher gelegenen Seitentälern interpretiert werden kann. Als größte Siedlung etablierte sich in dieser Zeit die die 3,5 ha großen Siedlung Donje Moštre, die um 4650 v. u. Z. im nördlichen Teil des Visokobeckens Okolište ablöste und bis etwa 4300 v. u. Z. bestand.

Ab 4500 v. u. Z. deutet sich ein Rückgang der Besiedlungsdichte in Zentralbosnien an, der allerdings teilweise dem noch unzureichenden Forschungsstand $\mathrm{zu}$ dieser Periode geschuldet sein kann. Dass man diesen Trend durchaus ernst nehmen darf, zeigt sich allerdings daran, dass trotz intensiver archäologischer Forschung für die Periode zwischen 4300 und 3600 v. u. Z. in Zentralbosnien kaum Fundplätze bekannt sind. Eine substanzielle Zunahme der Bevölkerungsdichte ist erst wieder mit dem Beginn der Badener Kultur feststellbar.

\section{Sozialhistorische Interpretationen}

Aus architektursoziologischer Perspektive sprechen die in Okolište und anderen Fundplätzen festgestellten regelmäßigen Siedlungsstrukturen für eine sehr geplante Vorgehensweise bei der Anlage der Siedlungen, die unter anderem im Rahmen von bewussten Ortsgründungen erfolgt sein könnte. Die Siedlungspläne zeichnen sich - gesprochen in Termini der von Bill Hillier und Julienne Hanson entwickelten space syntax theory - durch ein hohes Maß an ,Axialität' und einen geringen Grad an ,Konvexität' aus, was für eine soziale Verteilung räumlicher Kontrolle und ein hohes Maß an gegenseitiger sozialer Kontrolle spricht. ${ }^{29}$ Demnach müsste in der spätneolithischen Gesellschaft Zentralbosniens eine Ideologie geherrscht haben, in der das Dorf als Ganzes Bezugspunkt aller Sozialbeziehungen war.

Da es empirischen ethnographischen Parallelen zufolge nicht wahrscheinlich ist, dass eine Siedlung in der Größenordnung wie Okolište ohne Führung ausgekommen ist, wurde die Frage nach den sozio-politischen Organisationsstrukturen der spätneolithischen Gesellschaft sowohl auf der Ebene einzelner Siedlungen als auch im Maßstab der Siedlungskammer diskutiert. ${ }^{30}$ An Unterschieden von Siedlungsgrößen und der Existenz des Grabensystems kann auf regionaler Ebene zeitweise ein Primat der Siedlung Okolište über andere Orte abgeleitet werden, das sich im Laufe des Spätneolithikums zunehmend verliert und sich stärker zu einer Dichthomie zwischen größeren Dörfern in den Hauptflusstälern und kleinen Siedlungen in höheren peripheren Lagen wandelt. Im Rahmen dieses Prozesses verlor Okolište nach und nach seine Einwohner.

Anhand von Artefaktverteilungen in der größten zusammenhängenden Grabungsfläche 3 in Okolište ließen sich Unterschiede zwischen den Inventaren von Hausstellen nachweisen, die auf Unterschiede in der ökonomischen Aktivi-

\footnotetext{
${ }^{29}$ Müller-Scheeßel u. a. 2010a.

${ }^{30}$ Hofmann u. a. 2010 b.
} 
tät von Häusern hinweisen und als Hinweis auf die Existenz wirtschaftlich und rituell besonders aktiver Haushalte interpretiert werden (Abb. 6 b). ${ }^{31}$ Diese Haushalte gehören zu Gruppen von Hausstellen, für die eine ähnliche Verzierungsornamentik postuliert wurde. Die Basis der Sozialökonomie der Siedlung Okolište könnte dem daraus entwickelten Modell zufolge ein kleinräumiges Interagieren von Personengruppen in Gruppen zusammengehöriger Haushalte dargestellt haben, zwischen denen ein reziproker Austausch von Gütern stattfand. In den Mitgliedern der wirtschaftlich besonders aktiven Häuser wird diejenige Personengruppe vermutet, welche die kommunalen Belange regelte.

Dass die potentiell aus einer Überproduktion resultierende politische Macht möglicherweise auch über den Maßstab einzelner Siedlungen hinaus auf regionale Ebene gereicht haben könnte, deutet sich an Unterschieden des agrarischen Potentials und der Inventare einiger Fundplätze an. ${ }^{32}$ Alternativ kann man entsprechende Unterschiede zwischen den Fundinventaren von Siedlungen allerdings auch im Sinne einer sich entwickelnden wirtschaftlichen Diversifizierung bzw. kommunalen Spezialisierung verstehen, die auf Unterschieden im wirtschaftlichen Potential von Siedlungsumfeldern gründet.

Interkulturellen Vergleiche zufolge besteht generell ein Zusammenhang zwischen der Grundfläche und der Bewohnerzahl von Häusern. ${ }^{33}$ Demnach gibt die Größe von Häusern tendenziell einen Hinweis auf den Umfang der Familien, die in den Häusern lebten. Die mehrheitlich relativ kleinen Grundflächen der Häuser von Okolište zwischen 30 und $50 \mathrm{~m}^{2}$ und ihre durchschnittlich relativ kurze Lebensdauer von ca. 30 Jahren im-

\footnotetext{
${ }^{31}$ Müller u. a. 2011, Müller u. a. 2013c. Als „Hausstellen“ werden in diesen Beiträgen die Häuser zuzüglich der sie umgebenden Areale verstanden. Bei der Zuweisung der Funde zu konkreten Hausstellen wurde von der Annahme ausgegangen, dass der Abfall der Häuser mehrheitlich in ihrem unmittelbaren Umfeld entsorgt wurde und dort verblieb. Damit wird zumindest theoretisch die Schwierigkeit umgangen, dass die Inventare der Häuser selbst nachweislich in hohem Maße durch Depositionsprozesse (z. B. Ausräumung, Überlagerung) beeinträchtigt sind (s.o.; Hofmann 2013, 253 ff.). Die „Inventare“ beziehen sich durch dieses Verfahren nicht auf einzelne Häuser, sondern auf „Hausstellen“, die in der Regel zwei bis drei „Hausgenerationen" umfassen.

${ }^{32}$ Hofmann u. a. 2010b; Furholt 2012, 2013.

${ }^{33}$ Z. B. Naroll 1962; Wiessner 1974; Brown 1987; Porčić 2011.
}

plizieren, dass sie von sog. Kern- oder Kleinfamilien bewohnt waren und mit der Gründung einer solchen Familie in der Regel der Bau eines neuen Hauses einherging. Fraglich ist allerdings, ob die Häuser mit Haushalten gleichgesetzt werden dürfen, oder mehrere Häuser verwandtschaftlich und ökonomisch verbunden waren.

Darauf, dass möglicherweise mehrere benachbarte Häuser zu einen Haushalt gehörten, verweisen unter anderem unterschiedliche Erhaltungszustände von Häusern: Vielfach lagen Gebäude, die am Ende ihrer Nutzungszeit vollständig verbrannt waren, neben solchen, die bereits etwas früher aufgelassen worden waren. ${ }^{34}$ Deutlich wird dies abgesehen von Fläche 3 auch im Nordosten der Siedlung, wo im Plan der Geomagnetik verbrannte Häuser mit regelmäßigen Abständen sichtbar sind (Abb. 3). Die Ausgrabungen in Fläche 4 belegen, dass zwischen diesen verbrannten Gebäuden Reste unverbrannter Häuser lagen, die bereits vor dem Siedlungsbrand verfallen waren und deren Areale teilweise zur Abfallentsorgung der zuletzt bestehenden Häuser genutzt worden waren. In Verbindung mit der relativ kurzen, etwa eine Generation währenden Nutzungsdauer der Häuser könnte man dieses Muster im Sinne einer Generationenabfolge deuten. Demnach muss man in Betracht ziehen, dass mehrere Generationen (derselben Familie?) benachbarte Gebäude bewohnten und gemeinsame Haushalte bildeten.

Anhand der räumlichen Verteilung von Artefakten in Fläche 3, lässt sich diese Interpretation derzeit nicht schlüssig untermauern: Da jedes Haus im Laufe seiner Biographie eine aktive Periode „durchlebt“ haben müsste, als dessen Bewohner jung waren, wäre über eine Dauer von zwei bis drei Hausgenerationen eher eine gleichmäßige Verteilung von Aktivitätsindikatoren zu erwarten. Dies scheint nicht der Fall zu sein. Als Indikatoren für Jagd interpretierten Häufungen von Pfeilspitzen in einigen potentiell unterversorgten Hausstellen sind zudem kaum mit „altersgerechten" Aktivitätsprofilen vereinbar.

Ab ca. 4800 v. u. Z. ist in Okolište und auch in anderen Siedlungen Zentralbosniens ein klarer Trend zu größeren Häusern erkennbar. Angesichts der möglichen Zusammengehörigkeit von

\footnotetext{
${ }^{34}$ Entsprechende Muster wurden in den Grabungsflächen 1, 3 und 4 nachgewiesen (Hofmann 2013, 81 ff., 98 ff., 107 ff.).
} 
Häusern bereits in früheren Siedlungsphasen impliziert dies allerdings nicht unbedingt eine Vergrößerung von Haushalten, sondern könnte auch eine Zusammenlegung bisher baulich separierter Haushalts-Segmente anzeigen. Es scheint also eine stärkere interne Integration von Haushalten stattgefunden zu haben. Neue bauliche Formen für Mehrgenerationen-Haushalte aus der Zeit zwischen 4400 und 4300 v. u. Z. wurden in Donje Moštre nachgewiesen. Analog zu Hausformen im östlichen Karpatenbecken und dem mittleren Donaugebiet bestanden hier große (30 m lange), in Kompartimente aufgeteilte Gebäude, in denen mehrere Kernfamilien zusammen lebten und die bei Bedarf erweitert wurden. ${ }^{35}$

Auf die technologische und stilistische Entwicklung von Gefäßkeramik ist weiter oben bereits verwiesen worden. Die Variabilität der Keramik ist ganz offensichtlich durch die lokale und regionale Entwicklungsdynamik einer Gesellschaft mit stark vergrößerten sozialen Gruppen (Orten) und den daraus resultierenden sozialen und ökonomischen Veränderungen determiniert: Für die Entstehung so großer Orte wie Okolište war offenbar ein ausgeprägtes demographisches Wachstum ausschlaggebend; die Entwicklung von Keramikstilen reflektiert variable Intensitäten sozialer Interaktion innerhalb der spätneolithischen Gesellschaft.

Dass in der Phase zwischen 5000 und 4500 v. u. Z. einerseits Keramikstile in Südosteuropa durch eine ausgeprägte Regionalisierung gekennzeichnet sind und andererseits ausgeprägte Bevölkerungskonzentrationen in teils ausgedehnten Dörfern bestanden, dürfte eng zusammen hängen. Als sich am Übergang zur frühen Kupferzeit die differenzierten Siedlungssysteme auflösten und möglicherweise ein genereller Bevölkerungsrückgang stattfand, bildeten sich wieder großräumiger verbreitete Keramikstile heraus. Verschiebungen kultureller Grenzen und unterschiedliche Grade der Regionalisierung von Keramikstilen erklären sich demnach maßgeblich durch die Konfiguration von Siedlungssystemen; größere Wanderungsbewegungen sind hingegen aus der Sicht des Autors als Erklärungsmodelle der beobachteten Variabilität materieller Kultur nicht unbedingt notwendig.

\footnotetext{
${ }^{35}$ Hofmann / Müller-Scheeßel 2013a.
}

Für den Niedergang der entwickelten spätneolithischen Siedlungssysteme mit langlebigen Tells und Großsiedlungen spielen sicherlich mehrere Faktoren eine bedeutende Rolle. ${ }^{36}$ In Zentralbosnien erweist sich die Umstrukturierung des Siedlungssystems als ein langfristiger Prozess, der spätestens um 4850 v. u. Z. mit der zweiten Verkleinerung der Großsiedlung Okolište einsetzte und bis etwa 4300 v. u. Z. andauerte. Wir vermuten insbesondere sozioökonomische Gründe für den Niedergang: Überproduktion einiger Haushalte und Unterversorgung anderer scheint zur Entstehung sozialer Ungleichheit und zur zunehmenden Ungleichverteilung politischer Macht geführt haben. Dies dürfte die Suche benachteiligter Bevölkerungssegmente nach ökonomischen Nischen und damit die Aufspaltung der Gesellschaft in kleinere soziale Gruppen forciert haben. Weitere Faktoren dürften die Minderung der Lebensqualität durch die zunehmende Verschmutzung der eng bebauten Orte und die Separation vieler junger Männer von der Dorfgemeinschaft im Zusammenhang mit transhumanten Weideaktivitäten dargestellt haben. ${ }^{37}$

\section{Wirtschafts- und Landschaftsarchäologische Untersuchungen}

Die Analyse verkohlter botanischer Makroreste durch Dušanka Kučan (Wilhelmshaven) und Helmut Kroll (Kiel) belegen die Kultivierung und die Nutzung eines breiten Spektrums an Kulturund Sammelpflanzen. ${ }^{38}$ Einkorn und Emmer waren die wichtigsten Getreidesorten, neben denen unterschiedliche Hülsenfrüchte (z. B. Linse, Erbse) angebaut wurden. Lein diente als Lieferant für Öl und Flachs für die Herstellung von Kleidung. Funde von Hasel und Kornelkirsche waren derart häufig, dass Helmut Kroll von einer gezielten Schonung und Pflege in Hainen als Vorform der Kultivierung ausgeht. Als psychoaktive Pflanzen sind Bilsenkraut und Schwarzer Nachtschatten belegt.

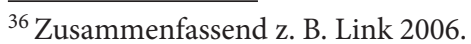

${ }^{37}$ Müller-Scheeßel u. a. 2010c; Müller-Scheeßel u. a. 2010b.

${ }^{38}$ Kučan 2006; Kroll 2013a, Kroll 2013b, Kroll in Vorbereitung.
} 
Eine nahezu das gesamte Neolithikum umfassende Siedlungssequenz mit umfangreichen Funden an Tierknochen liegt von den beiden Fundstellen Obre Gornje Polje und Obre Raskršće vor, die in den 1960er Jahren durch ein bosnisch-amerikanisches Team ausgegraben wurden. ${ }^{39}$ Die früh- und mittelneolithischen Schichten (5700-5200/5000 v. u. Z.) dieser Siedlungen sind durch relativ ausgeglichene Anteile von Schafen/Ziegen einerseits und Rindern andererseits gekennzeichnet. ${ }^{40} \mathrm{Im}$ Spätneolithikum (5200/5000-4300 v. u. Z.) ist dann eine deutliche Zunahme der Bedeutung von Rinderhaltung und ein Rückgang der Anteile von Wildtieren zu beobachten.

Leider erweist sich die Erhaltung von Tierknochen in den Siedlungen, die im Rahmen des aktuellen Projektes untersucht wurden, größtenteils als schlecht. Für die Periode nach 5200/5000 v. u. Z. bestätigte sich die Dominanz von Rinderhaltung die im Verlauf des Spätneolithikums und frühen Äneolithikums noch zunimmt. ${ }^{41}$ Jagt spielte in den untersuchten Siedlungen eine noch geringere Rolle als in Obre Gornje Polje. Die zunehmende Spezialisierung auf Rinderhaltung im Verlauf des Neolithikums ist in Südosteuropa ein großräumiges Phänomen, das als mögliches Resultat vergrößerter Herden (vielleicht als soziales Kapital) gesehen wird und eine zunehmende Separierung der Weide von anderen Aktivitäten erforderte. ${ }^{42}$

Vergleiche des Kalorienbedarfs der rekonstruierten Bevölkerungsgrößen mit der carrying capacity der Siedlungsumfelder führten zu der Hypothese, dass sich der Wirtschaftsraum der neolithischen Siedlungen kaum auf die Flusstäler Zentralbosniens beschränkt haben dürfte, sondern auch bereits höhere Gebirgslagen für die Viehweide genutzt wurden. ${ }^{43}$ In einem Pollenprofil aus dem in $1670 \mathrm{~m}$ über dem Meeresspiegel im Vranica-Gebirge gelegenen Prokoško Jezero, das von der Arbeitsgruppe Walter Dörflers (Kiel) $\mathrm{zu}$ Test dieser Hypothese analysiert wurde, ist ab ca. 5500 menschlicher Einfluss sichtbar, der auf eine Nutzung des Gebirges als Viehweide bestä-

\footnotetext{
${ }^{39}$ Benac 1973a; Benac 1973b; Gimbutas 1974a; Gimbutas 1974b; Sterud / Sterud 1974.

${ }^{40}$ Bököny 1974.

${ }^{41}$ Benecke 2006; Benecke 2013; Bultmann 2010.

${ }^{42}$ Orton 2012.

${ }^{43}$ Müller 2006.
}

tigt. ${ }^{44}$ Mit der jahreszeitlichen Nutzung höherer Gebirgslagen ging vermutlich eine Separierung eines Bevölkerungssegmentes (junge Männer) einher, die desintegrative Auswirkungen auf das soziale Gefüge spätneolithischer Gesellschaften besessen haben dürfte und damit einen Faktor für den Niedergang des spätneolithischen Siedlungssystem dargestellt haben könnte. ${ }^{45}$

Innerhalb des Visokobeckens weisen einige Siedlungsumfelder möglichweise zu geringe Ackerflächen auf, um den Kalorienbedarf der Bevölkerungsgrößen zu decken, die basierend auf Anzahl und Größe von Häusern sowie Bebauungsdichten geschätzt wurden. Zudem bestehen zwischen einigen Siedlungen Unterschiede in der Häufigkeit bestimmter Fundkategorien, die auf gegenseitige Abhängigkeiten zwischen Orten bzw. Spezialisierungen auf bestimmte Wirtschaftszweige hinweisen könnten. Beispielsweise war in Kundruci eine außerordentlich geringe Dichte an Mahlsteinen und Getreideunkräutern festzustellen. ${ }^{46}$ Zugleich weisen die Siedlungsschichten eine wesentlich höhere Konzentration von Phosphor als jene in Donje Moštre auf. ${ }^{47}$ Möglichweise war die Bevölkerung in Kundruci demnach stärker auf Viehzucht spezialisiert als die Dörfer im Haupttal der Bosna.

Südöstlich von Kakanj, etwa $3 \mathrm{~km}$ östlich der Siedlungen Obre Raskršće und Obre Gornje Polje, wurde durch Steffen Wolters und Felix Bittmann (Wilhelmshaven) ein Pollenprofil aus dem Niedermoor Seoce Jezero untersucht, dessen Sedimentation in der frühen Bronzezeit einsetzte. ${ }^{48}$ Auch Pollenproben aus der Sohle unmittelbar an den Fundplatz Okolište angrenzenden Altarm der Bosna erwiesen sich als bronzezeitlich. ${ }^{49}$ Geomorphologische Untersuchungen an unterschiedlichen Standorten im Tal der Bosna erlauben die Synchronisierung verstärkter Rodungen im Gebirge mit massiven Änderungen der Flussaktivität der Bosna in Form verstärkter Erosion, erhöhten Wasserabflussmengen und Hochwasserereignissen seit der Bronzezeit. $^{50}$ Seit dem frühen 2. Jahrtausend v. u. Z. sind also

\footnotetext{
${ }^{44}$ Dörfler 2013.

${ }^{45}$ Müller Scheeßel u. a. 2010 b.

${ }^{46}$ Furholt 2013.

${ }^{47}$ Dreibrodt u. a. 2013a; Dreibrodt u. a. 2013b.

${ }^{48}$ Wolters / Bittmann 2006.

${ }^{49}$ Dörfler 2006.

${ }^{50}$ Dreibrodt u. a. 2013a; Dreibrodt u. a. 2013b.
} 
erhebliche Landschaftsveränderungen und eine generelle Intensivierung der Siedlungstätigkeit im Einzugsgebiet der Bosna belegt. Indirekt implizieren diese Ergebnisse für das Neolithikum und die Kupferzeit eine deutlich geringere Flussaktivität der Bosna und eine geringere Öffnung der Landschaft. Als Reste dieser frühholozänen Landschaft sind bis heute im Gelände südlich von Okolište weitläufige, stark verflachte Mäander sichtbar. ${ }^{51}$ Knochen von Bibern, karpfenartigen Fischen, Hechten und Graureihern belegen ein fischreiches, langsam fließendes Gewässer. ${ }^{52}$

Anthrakologische Untersuchungen an Holzkohlen aus den Grabungen durch Tim Schroedter (Kiel) zeigen die Nutzung unterschiedlicher ökologische Zonen im Umfeld der Siedlungen. ${ }^{53}$ Während des Spätneolithikums fand im Visokobecken eine zunehmende Öffnung der Landschaft statt, die jedoch offenbar zunächst nicht zu einschneidenden Änderungen der Flussaktivität führte. Nach 4300 v. u. Z. zeichnet sich an spätneolithischen Siedlungsstandorten eine Wiederbewaldung ab. ${ }^{54}$

\section{Zusammenfassung}

Das Ziel eines mittlerweile abgeschlossenen bosnisch-deutschen Forschungsprojektes bestand darin, die Aussiedlung des bosnischen Mittelgebirges durch Gesellschaften mit neolithischer Wirtschaftsweise, die damit verbundene Siedlungsdynamik und die sozialen, demographischen und ökonomischen Rahmenbedingungen dieses Prozesses für einen Zeitraum zwischen ca. 5800-4300 v. u. Z. möglichst umfassend $\mathrm{zu}$ rekonstruieren. Im Fokus dieser Forschungen stand die spätneolithische Großsiedlung Okolište aus der Zeit zwischen 5200/5100 und 4700 v. u. Z.

Die zentralbosnische Mittelgebirgszone bildete im Neolithikum den westlichsten Ausläufer der balkanischen Tellkulturen, die durch differenzierte Siedlungssysteme mit teils großen zentralen Dörfern gekennzeichnet sind. In Zentralbosnien waren die Dynamik und die kulturelle

\footnotetext{
${ }^{51}$ Hofmann 2013

52 Bultmann 2010

${ }^{53}$ Schroedter 2009; Schroedter u. a. 2012; Schroedter / Nelle 2013.

${ }^{54}$ Dreibrodt u. a. 2013a; Dreibrodt u. a. 2013b.
}

Ausprägung der hier lebenden Gesellschaften in starkem Maße durch die kulturgeographische Lage der Region zwischen dem Ostadriagebiet einerseits und dem zentralen Balkangebiet andererseits determiniert. Mit variierenden Intensitäten bestanden während des gesamten Neolithikums Kommuni-kationsbeziehungen in beide Regionen.

Wichtig erscheint, dass zwischen regionaler und lokaler Siedlungsentwicklung einerseits und stilistischer und technologischer Entwicklung der Gefäßkeramik andererseits Zusammenhänge zu bestehen scheinen: Die Entstehung von „Butmir"-Keramik im Verständnis von Alois Benac basiert demnach auf einer zeitweise erhöhten Intensität überregionaler Kontakte und damit verbundener stark erhöhter Innovativität. Zugrunde liegt ein schnelles Bevölkerungswachstum, dessen Höhepunkt wohl in der Periode zwischen 5500 und 5200 v. u. Z. lag. Dieses demographische Wachstum führte um $5200 \mathrm{v}$. u. Z. zur Gründung der Großsiedlung Okolište und später nach und nach zur weiteren Aufsiedlung des zentralbosnischen Raumes.

Dieser Prozess ging mit Änderungen der Wirtschaftsweise einher: Rinderhaltung wurde intensiviert und man begann, höhere Gebirgslagen für saisonale Viehhaltung zu nutzen. Bestände von Sammelpflanzen wie Hasel und Kornelkirsche wurden in Hainen gepflegt. Es kam zu einer Auflichtung der Flusstäler.

Wir gehen davon aus, dass die Konzentration von bis zu mehreren Tausend Menschen in dem Dorf Okolište völlig neuartige soziale Probleme aufwarf und zur Entstehung neuer politischer Institutionen führte, die als zusätzliche Mechanismen zur Bewältigung höherer sozialer Komplexität fungierten. Dem Ideal der Reziprozität ökonomischer Beziehungen zwischen unabhängigen Haushalten stand potenziell eine Realität gegenüber, in der einzelne Individuen bzw. Haushalte durch eine Überproduktion von Subsistenzgütern politische und rituelle Macht erlangten. Diese könnte die Basis für die Entstehung sozialer Ungleichheit und ein maßgeblicher Faktor für die Aufspaltung der zeitweise bis mehrere tausend Menschen umfassenden Dorfgemeinschaften in deutlich kleinere soziale Gruppen dargestellt haben.

In den archäologischen Quellen äußern sich die skizzierten Beziehungen zwischen Haus- 
halten einerseits in Inventarunterschieden von Hausstellen und andererseits in einer zeitweise deutlich erhöhten Diversität und Repräsentativität der Gefäßkeramik. Insgesamt implizieren die genannten Schlussfolgerungen, dass die rekonstruierte Siedlungsdynamik und die Ausprägung der materiellen Kultur maßgeblich durch regionale, gesellschaftsinterne soziale und ökonomische Prozesse erklärbar sind. Welche Rolle äußere, überregionale Faktoren spielten, ist dagegen derzeit schwerer einschätzbar.

Die genannte Deutung erhält durch die Entwicklung in der Zeit ab ca. 4850 v. u. Z. zusätzliche Plausibilität: Einhergehend mit der sukzessiven Transformation des regionalen Siedlungssystems, das nun in geringerem Maße durch große Bevölkerungskonzentrationen geprägt ist, sind die Komplexitätsmarker der Keramik rückläufig. Im Gegenzug wird eine stärker überregionale Orientierung der Keramikstile sichtbar, die wiederum auf eine intensivere Kommunikation mit unterschiedlichen Nachbarregionen hindeutet. Vor dem Hintergrund erheblich kleinerer Siedlungen könnte die Notwendigkeit zu exogamen Heiraten ein möglicher Faktor für diese Entwicklung sein.

In der Endphase des Spätneolithikums und im frühen Äneolithikum werden die Beziehungen in das zentrale Balkangebiet dominant. Neben Keramikstilen mit Kanneluren äußert sich dies unter anderem an deutlich größeren Häusern in Donje Moštre, die ähnlich wie im Donauraum und in Ostungarn aus mehreren Räumen zusammengesetzt sind, die jeweils über eine Feuerstelle verfügen. Kurz danach, um 4300 v. u. Z., endet schließlich die Siedlungsweise, die zur Akkumulation von Siedlungshügeln führte.

\section{Rezime}

\section{Okolište - razvoj keramike i naselja iz kasnog neolita na području srednje Bosne: rezultati i interpretacije}

Nedavno završeni bosansko-njemački istraživački projekat imao je za cilj što obuhvatniju rekonstrukciju neolitskog naseobinskog sistema u bosanskom srednjogorju, tj. razmatranje dinamike naseljavanja te sagledavanje socijalnih, demografskih i ekonomskih okvira tog procesa u razdoblju od 5800. do 4300. godine pr. n. e. Polazna tačka istraživanja bilo je veliko neolitsko naselje Okolište iz vremena 5200/51004700. pr. n. e.

Zona centralnobosanskog srednjogorja u neolitu je predstavljala zapadni ogranak balkanskih tell-kultura koje karakteriše izdiferencirani sistem naseljavanja, najčešće $s$ velikim centralnim naseljima. Dinamika i kulturni izraz neolitskih zajednica s prostora centralne Bosne u znatnoj mjeri su determinisani geo-kulturnim položajem regiona između jadranskog područja s jedne strane i srednjeg Balkana s druge strane. Na ovim relacijama su tokom čitavog neolita postojale komunikacije različitog intenziteta.

Značajno je da su na regionalnom i na lokalnom nivou izraženi zajednički elementi u razvoju naselja s jedne strane i stilističkog i tehnološkog razvoja keramičkog posuđa s druge strane. Kako navodi Alojz Benac, nastanak „butmirske“ keramike je bio uslovljen pojačanim intenzitetom međuregionalnih kontakata i naglašenom inovativnošću koja je tome slijedila. Osnova svega je bio relativno brzi porast stanovništva koji dostiže svoj vrhunac u dobu između 5500. i 5200. godine pr. n. e. Demografski uspon doveo je i do osnivanja velikog naselja u Okolištu oko 5200. pr. n. e., čemu je slijedilo dalje naseljavanje područja srednje Bosne.

Taj proces su pratile promjene u načinu privređivanja: intenziviranje govedarstva i početak korištenja visinskih pašnjaka za sezonsko stočarstvo. Uz to se pospješuje uzrast lješnika i koštunica u okolnim šumarcima, a riječne doline se krče i raščišćavaju.

Smatramo da je koncentracija od nekoliko hiljada ljudi, koliko je živjelo u naselju Okolište, indukovala sasvim nove društvene probleme, a time i uzrokovala nastanak novih političkih institucija i mehanizama, koji su bili regulatori pojačane kompleksnosti društva. Idealnom reciprocitetu ekonomskih odnosa između nezavisnih domaćinstava suprostavlja se nova realnost, u kojoj pojedine individue, odnosno domaćinstva, otpočinju sa stvaranjem viška supsistencijalne proizvodnje, čime se stiče pozicija za osvajanje političke i ritualne moći. To je moglo biti uzrok za nastanak socijalne nejednakosti i postati odlučujuću faktor u razbijanju velike naseobinske zajednice i u njenom dijeljenju na više znatno manjih grupa.

U arheološkim izvorima ti se odnosi iskazuju u razlikama inventara među domaćinstvima, kao i u djelimično snažno povećanoj diversifikaciji i reprezentativnosti keramičkog posuđa. Navedeni zaključci u cjelini upućuju na to da se rekonstruisana dinamika naseljavanja, kao i specifične karakteristike materijalne kulture, u znatnoj mjeri mogu objasniti na osnovu karakteristika regionalnih, odnosno internih socijal- 
nih i ekonomskih procesa. Nasuprot tome, mnogo je teže razlučiti kakvu ulogu su tu imali spoljni, nadregionalni faktori. Razvoj od ca. 4850. godine pr. n. e još više potvrđuje vjerodostojnost navedenih postavki. Paralelno sa sukcesivnom transformacijom regionalnog sistema naseljavanja koji je sve manje okarakterisan velikim koncentracijama stanovništva, smanjuje se i kompleksnost keramike. Sada je primijetna veća nadregionalna usmjerenost keramičkog stila, što opet upućuje na intenziviranje komunikacija s raznim susjednim oblastima. Polazeći od znatno smanjenih naselja, može se pretpostaviti da je i potreba za egzogamnim brakom bila jedan od mogućih faktora takvog razvoja. U zadnjoj fazi kasnog neolita i u ranom eneolitu dominantne su veze s centralnim Balkanom. Pored kanelovane keramike, to je vidljivo i u pojavi znatno većih kuća u Donjim Moštrama koje, poput onih iz Podunavlja i iz istočne Mađarske, sadrže više prostorija od kojih svaka ima ognjište. Kratko nakon toga, oko 4300. pr. n. e. socio-ekonomski sistem o kome smo govorili i koji je doveo do akumulacije tellnaselja, u potpunosti nestaje.

\section{Literaturverzeichnis}

Benac, A. 1973 a, Obre I - A Neolithic Settlement of the Starčevo-Impresso and Kakanj Cultures at Raskršće, Wissenschaftliche Mitteilungen des Bosnisch-Herzegowinischen Landesmuseums 3 A, 1973, 327-429.

Benac, A. 1973 b, Obre II - A Neolithic settlement of the Butmir Group at Gornje Polje, Wissenschaftliche Mitteilungen des Bosnisch-Herzegowinischen Landesmuseums 3 A, 1973, 5-325.

Benac, A. 1979, Prelazna Zona, In: Basler u. a. 1979 , 363-470.

Benecke, N. 2006, Archäozoologische Untersuchungen, In: Hofmann u. a. 2006, 159-161.

Benecke, N. 2013, Archäozoologische Untersuchungen - Arbeitsbericht 2008, In: Müller u. a. 2013a 239-240.

Bökönyi, S. 1974, The vertebratefauna, Wissenschaftliche Mitteilungen des Bosnisch-Herzegowinischen Landesmuseums A 4, 1974, 55-154.

Brown, B. M. 1987, Population Estimationfrom Floor Area: A Restudy of "Naroll's Constant", Behavior Science Research 21, 1987, 1-49.

Bultmann, U. 2010, Landschaft, Tiere, Analysen. Tierknochenanalysen und Landschaftsnutzung butmirzeitlicher Fundplätze im Visokobecken $(\mathrm{BiH})$ (Diplomarbeit Universität Kiel 2010).
Bultmann, U. 2012, Putting sites in their catchment areas. In: Hofmann u. a. 2012, 221-230.

Dörfler, W. 2006, Hinweise zur Umwelt aus einem Altarm der Bosna bei Okolište, In: Hofmann u. a. 2006, 175-176.

Dörfler, W. 2013, Prokoško Jezero: an environmental record from a subalpine lake in Bosnia-Herzegovina, In: Müller u. a. 2013a, 311-340.

Dreibrodt u. a. 2013a: Dreibrodt, St. / Hofmann, R. / Lubos, C. / Dazert, S. / Zahrer, J. / Fuchs, M. / Bork, H.-R. 2013, Ergebnisse geomorphologisch-geoarchaologischer Begleituntersuchungen an den Siedlungen im Visokobecken (Bosnien-Herzegowina), In: Müller u. a. 2013a, 287-310.

Dreibrodt u. a. 2013b: / Müller-Scheeßel, N. /Richling, I. / Nelle, O. / Fuchs, M. / Rassmann, K. / Kujundžić-Vejzagić, Z. / Bork, H.-R. / Müller, J. 2013, Holoceneri verandslopeactivity in the Visoko Basin, Bosnia-Herzegovina - Climate and landuse effects, Journal of Quaternary Science 28, 6, 2013, 559-570.

Furholt, M. 2012, Kundruci: Development of socialspace in a Late Neolithic Tell settlement in Central Bosnia, In: Hofmann u. a. 2012, 203-219.

Furholt, M. 2013, Abseits des Weges - Prospektionen und Ausgrabungen in Kundruci, In: Müller u. a. 2013a, 173-206.

Gimbutas, M. 1974a, Introduction. Obre and its place in Old Europe, Wissenschaftliche Mitteilungen des Bosnisch-Herzegowinischen Landesmuseums A 4, 1974, 5-13.

Gimbutas, M. 1974b, Chronology of Obre I and II, Wissenschaftliche Mitteilungen des Bosnisch-Herzegowinischen Landesmuseums A 4, 1974, 15-35.

Herrmann, u. a. 1990: Herrmann, B. / Gruppe, G. / Hummel, S. / Piepenbrink, H. / Schutkowski, H. 1990, Prahistorische Anthropologie. Leitfaden der Feld und Labormethoden, Berlin, Heidelberg, New York 1990.

Hofmann R. 2013, Okolište 2 - Spätneolithische Keramik und Siedlungsentwicklung in Zentralbosnien, Neolithikum und Chalkolithikum in Zentralbosnien 2, Universitätsforschungen zur prähistorischen Archäologie 243, Bonn 2013.

Hofmann, R. / Hofmann, A. 2013, Anthropomorphe und zoomorphe Figurinen des Spätneolithikums aus Okolište und der Butmirgruppe in Zentralbosnien, Darstellungsmodi, Herstellungstechniken, Datierungen, Häufigkeiten und Fundkontexte. Offa 69/70, 2012/13, 439-459.

Hofmann, R. / Müller-Scheeßel, N. 2013a, Der kupferzeitliche Fundplatz Donje Moštre bei Visoko in Zentralbosnien - Ein Vorbericht, In: Müller u. a. 2013a, 207-229.

Hofmann, R. / Müller-Scheeßel, N. 2013b, Der spätneolithische Tell von Okolište im Visokobecken in 
Zentralbosnien: Ergebnisse der Grabungskampagnen 2006 und 2007, In: Müller u. a. 2013a, 69-102.

Hofmann, R. / Müller-Scheeßel, N. 2013c, Bericht über die Prospektionskampagne im Frühjahr 2008, In: Müller u. a. 2013a, 127-141.

Hofmann u.a. 2006: Hofmann, R. / Kujundžić-Vejzagić, Z. / Müller, J. / Müller-Scheeßel, N. / Rassmann, K. 2006, Prospektionen und Ausgrabungenin kolište (Bosnien-Herzegowina): Siedlungsarchaologische Studien zum zentralbosnischen Spatneolithikum (5300-4500 v. Chr.), Bericht der Romisch-Germanischen Kommission 87, 2006, 41-212.

Hofmann u. a. 2008: Hofmann, R. / KujundžićVejzagić, Z. / Müller, J. / Müller-Scheeßel, N. / Rassmann, K. 2008, Excavations in Okolište and the reconstruction of Late Neolithic settlement processes 41 in the Visoko Basin in Central Bosnia (5200-4500 B.C.), www.aegeobalkanprehistory. net, Beitrag vom 11. April 2008 [09.03.2012].

Hofmann u. a. 2008/09: Hofmann, R. / KujundžićVejzagić, Z. / Müller, J. / Rassmann, K. / MüllerScheeßel, N. 2008/09, Rekonstrukcija procesa naseljavana u kasnom neolitu na prostoru centralne Bosne, Glasnik Zemaljskog muzeja Bosne i Hercegovine u Sarajevu, Arheologija, Nova serija 50/51, 2008/09, 11-178.

Hofmann u. a. 2010a: Hofmann, R. / Müller-Scheeßel, N. / Müller, J. / Rassmann, K. 2010, Mittler zwischen den Welten - fruhe Tellkultur in Zentralbosnien, Archaologie in Deutschland 2010/4, 56-59.

Hofmann u. a. 2010b: Hofmann, R. / Müller-Scheeßel, N. / Müller, J. / Rassmann, K. 2010, Sozio-politische Organisationsstrukturen und zentrale Institutionen des spatneolithischen Visokobeckens in Zentralbosnien (5500-4500 v. Chr.), In: Hansen, S. (Hrsg.), Leben auf dem Tell als soziale Praxis, Beitrage des Internationalen Symposiums in Berlin vom 26.-27. Februar 2007, Kolloquien zur Vorund Fruhgeschichte 14, Bonn 2010, 189-213.

Hofmann U. a. 2012: Hofmann, R. / Moetz, F.-K. / Müller, J. (Hrsg.) 2012, Tells: Socialand Environmental Space, Proceedings of the International Workshop "Socio-Environmental Dynamics over the Last 12,000 Years: The Creation of Landscapes II (14th-18th March 2011)" in Kiel, Volume 3, Universitatsforschungen zur prahistorischen Archaologie 207, Bonn 2012.

Kroll, H. 2013a, Bericht über die Pflanzenfunde aus Okolište Haus 38, In: Müller u. a. 2013a, 113-122.

Kroll, H. 2013b, Die Wirtschaft des Umfelds von Okolište: Zagrebnice, Kundruci und Donje Moštre, In: Müller u. a. 2013a, 231-237.

Kroll in Vorbereitung: Kroll, H., Die Pflanzenfunde von Okolište, In: Müller, J. / Rassmann, K. (Hrsg.), Okolište - Umwelt, materielle Kulturund Gesellschaft in einer spätneolithischen und chalkoli- thischen Siedlungskammer in Zentralbosnien. Müller, J. / Rassmann, K. / Kujundžić-Vejzagić, Z. (Hrsg.), Neolithikum und Chalkolithikum in Zentralbosnien 3. Universitätsforschungen zur prähistorischen Archäologie, Bonn (in Vorbereitung).

Kučan, D. 2006, Vorläufiger Bericht über die Untersuchungverkohlter Pflanzenreste der spätneolithischen Siedlung Okolištein Bosnien, In: Hofmann u. a. 2006, 162-166.

Link Th. 2006, Das Ende der neolithischen Tellsiedlungen. Ein kulturgeschichtliches Phänomen des 5. Jahrtausends v. Chr. im Karpatenbecken, Universitätsforschungen zur prähistorischen Archäologie 134, Bonn 2006.

Müller, J. 1994, Das ostadriatische Frühneolithikum. Die Impresso-Kultur und die Neolithisierung des Adriaraumes, Prähistorische Archäologie in Südosteuropa 9, Berlin 1994.

Müller, J. 2006: J. Müller, Demographische Variablen des Bosnischen Spätneolithikums - Zur Frage der Bevölkerungsrekonstruktion im südosteuropäischen Neolithikum, In: N. Tasić / C. Grazdanov (Hrsg.), Homage to Milutin Garašanin, Belgrade 2006, 367-378.

Müller u. a. 2011: Müller, J. / Hofmann, R. / MüllerScheeßel, N. / Rassmann, K. 2011, Zur sozialen Organisation einer spätneolithischen Gesellschaft in Südosteuropa (5200-4500 v. Chr.), In: Hansen, S. / Müller, J. (Hrsg.), Sozialarchäologische Perspektiven: Gesellschaftlicher Wandel 5000-1500 v. Chr. zwischen Atlantik und Kaukasus, Internationale Tagung 15.-18. Oktober 2007 in Kiel, Archäologie in Eurasien 24, Darmstadt 2011, 81-106.

Müller u. a. 2013a: Müller, J. / Rassmann, K. / Hofmann, R. (Hrsg.), Okolište 1 - Untersuchungen einer spätneolithischen Siedlungskammer in Zentralbosnien. Neolithikum und Chalkolithikum in Zentralbosnien 1, Universitätsforschungen zur prähistorischen Archäologie 228, Bonn 2013.

Müller u. a. 2013b: Müller, J. / Rassmann, K. / Kujundžić-Vejzagić, Z. 2013, Okolište - Rekonstruktion spätneolithischer und frühchalkolithischer Siedlungsprozesse des zentralbosnischen Visokobeckens: Fragestellungen, Forschungsstrategien, Ergebnisse, In: Müller u. a. 2013a, 11-68.

Müller u. a. 2013c: Müller, J. / Hofmann, R. / MüllerScheeßel, N. / Rassmann, K. 2013, Neolithische Arbeitsteilung: Spezialisierung in einem Tell um 4900 v. Chr., In: Anders, A. / Kulcsár, G. (Hrsg.), Moments in Time, Papers presented to Pál Raczky on his 60th birthday, Prehistoric Studies, Budapest 2013, 407-420.

Müller-Scheeßel, N. 2013, Die geschlagenen Steinartefakteaus dem Visoko-Becken I - Die geschlagenen Steinartefakteaus Okolište, In: Müller u. a. 2013a, 241-286. 
Müller-Scheeßel, N. / Hofmann, R. 2013a, Der Fundplatz Zagrebnice bei Papratnica, In: Müller u. a. 2013a, 143-172.

Müller-Scheeßel, N. / Hofmann, R. 2013b, Dokumentation der Datenbank des Projektes „Rekonstruktion spätneolithischer Siedlungsprozesse in Zentralbosnien", In: Müller u. a. 2013a, 353-428.

Müller-Scheeßel u. a. 2007: Müller-Scheeßel, N. / Schmitz, J. / Hofmann, R. / Kujundžić-Vejzagić, Z. / Müller, J. / Rassmann, K. 2007, Die Totender spätneolithischen Tellsiedlung von Okolište / Bosnien-Herzegowina: Massaker, Seuche oder Bestattungsbrauch? www.jungsteinsite.de, Beitrag vom 15. Dezember 2007 [09.03.2012].

Müller-Scheeßel u. a. 2009: Müller-Scheeßel, N. / Schmitz, J. / Hofmann, R. / Kujundžić-Vejzagić, Z. / Müller, J. / Rassmann, K. 2009, Die Totender spätneolithischen Tellsiedlung Okolište / Bosnien-Herzegowina: Massaker, Seuche oder Bestattungsbrauch?, In: Zeeb-Lanz, A. (Hrsg.), Krisen - Kulturwandel - Kontinuitäten. Zum Ende der Bandkeramik in Mitteleuropa, Beiträge der internationalen Tagungin Herxheim bei Landau (Pfalz) vom 14.-17. 06. 2007. Internationale Archäologie. Arbeitsgemeinschaft, Symposium, Tagung, Kongress 10, Rahden / Westf. 2009, 327-340.

Müller-Scheeßel u. a. 2010a: Müller-Scheeßel, N. / Hofmann, R. / Müller, J. / Rassmann, K. 2010, Entwicklung und Struktur des spätneolithischen Tells von Okolište (Bosnien-Herzegowina) unter architektursoziologischen Gesichtspunkten, In: Trebsche, P. / Müller-Schee.el, N. / Reinhold, S. (Hrsg.), Der gebaute Raum. Bausteine einer Architektursoziologievormoderner Gesellschaften, Tübinger Archäologische Taschenbücher 17, Münster u. a. 2010, 171-192.

Müller-Scheeßel u. a. 2010b: Müller-Scheeßel, N. / Hofmann, R. / Müller, J. / Rassmann, K. 2010, The Socio-Political Development of the Late Neolithic Settlement of Okolište / Bosnia-Hercegovina: Devolution by Transhumance?, In: Kiel Graduate School „Human Development in Landscapes“ (Hrsg.), Landscapes and Human Development: The Contribution of European Archaeology, Proceedings ofthe International Workshop "SocioEnvironmental Dynamics over the last 12,000 Years: The Creation of Landscapes" (1st-4th April 2009), Universitäts forschungen zur prähistorischen Archäologie 191, Bonn 2010, 181-191.
Müller-Scheeßel u. a. 2010c: Müller-Scheeßel, N. / Hofmann, R. / Müller, J. / Rassmann, K. 2010, Müllmanagement in einem spätneolithischen Tell Zentralbosniens. Jahresschrift des Tübinger Vereinszur Förderung der ur- und frühgeschichtlichen Archäologie 11, 2010, 67-79.

Müller-Scheeßel u. a. 2013: Müller-Scheeßel, N. / Hofmann, R. / Rassmann, K./ Müller, J. 2013, OKOLISTE. Datenbank des Projektes „Rekonstruktion spätneolithischer Siedlungsprozesse in Zentralbosnien“, www.johanna-mestorf-academy.uni-kiel.de.

Naroll, R. 1962, Floor area and settlement population, American Antiquity 27, 1962, 587-588.

Orton, D. 2012, Herding, Settlement, and Chronology in the Balkan Neolithic, European Journal of Archaeology 15, 2012, 5-40.

Perić, S. 1995, Butmirska kultura. Geneza i razvoj. Butmir culture. Origin and development, Posebna izdanja Arheološki institut 29, Belgrad 1995.

Porčić, M. 2011, An exercise in archaeological demography: estimating the populations ize of Late Neolithics ettlements in the Central Balkans, Documenta Praehistorica 38, 2011, 323-332.

Schroedter, T. M. 2009, Holz und Holzbearbeitung in derneolithischen Siedlung Okolište, Bosnien-Herzegowina, Diplomarbeit, Universität Kiel 2009.

Schroedter, T. / Nelle, O. 2013, Untersuchungen von Holzkohlen aus Okolište - Ein Zwischenbericht, In: Müller u. a. 2013a, 123-126.

Schroedter u. a. 2012: Schroedter, T. M. / Hofmann, R. / Müller-Scheeßel, N. / Müller, J. / Nelle, O. 2012, Late Neolithic vegetation around three sites in the Visoko basin, Bosnia, based on archaeoanthracology - spatial variation versus selective wood use, In: Bedal, E. / Carrion, Y. / Macias, M. / Ntinou, M. (Hrsg.), Wood and Charcoal. Evidence for human and natural history, Sagvntvm. Papeles del Laboratorio de Arqueologia de Valencia, Extra 13, 2012, 53-64.

Sterud, E. L. / Sterud, A. K. 1974, A quantitative analysis of the material remains, Wissenschaftliche Mitteilungen des Bosnisch-Herzegowinischen Landesmuseums A 4, 1974, 155-355.

Wiessner, P. 1974, A Functional Estimation of Population from Floor Area, American Antiquity 39, 1974, 342-349.

Wolters, S. / Bittmann, F. 2006, Pollenanalytische Untersuchungen zur Landschaftsentwicklung im Visokobecken/Bosnien-Herzegowina, In: Hofmann u. a. 2006, 167-174. 
